\title{
Nucleon-nucleon scattering within a multiple subtractive renormalization approach
}

\author{
V. S. Timóteo, ${ }^{1}$ T. Frederico, ${ }^{2}$ A. Delfino, ${ }^{3}$ and Lauro Tomio ${ }^{3,4}$ \\ ${ }^{1}$ Faculdade de Tecnologia, Universidade Estadual de Campinas, 13484-332 Limeira, São Paulo, Brazil \\ ${ }^{2}$ Instituto Tecnológico de Aeronáutica, DCTA, 12228-900 São José dos Campos, São Paulo, Brazil \\ ${ }^{3}$ Instituto de Física, Universidade Federal Fluminense, 24210-346 Niterói, Rio de Janeiro, Brazil \\ ${ }^{4}$ Instituto de Física Teórica, UNESP-Universidade Estadual Paulista, 01140-070 São Paulo, São Paulo, Brazil
}

(Received 8 June 2010; revised manuscript received 8 March 2011; published 29 June 2011)

\begin{abstract}
We present a methodology to renormalize the nucleon-nucleon interaction in momentum space, using a recursive multiple subtraction approach that prescinds from a cutoff regularization, to construct the kernel of the scattering equation. The subtracted scattering equation is solved with the next-leading-order and nextto-next-leading-order interactions. The results are presented for all partial waves up to $j=2$, fitted to lowenergy experimental data. In this renormalization group invariant approach, the subtraction energy emerges as a renormalization scale and the momentum associated with it comes to be about the QCD scale $\left(\Lambda_{\mathrm{QCD}}\right)$, irrespectively to the partial wave.
\end{abstract}

DOI: 10.1103/PhysRevC.83.064005

PACS number(s): 21.30.-x, 03.65.Nk, 11.10.Gh, 13.75.Cs

\section{INTRODUCTION}

The nucleon-nucleon $(N N)$ interaction in leading order corresponds to the one-pion-exchange potential (OPEP) plus a Dirac $\delta$, when considering an effective field theory (EFT) of nuclear forces based on a chiral expansion of the effective Lagrangian. This procedure was suggested by Weinberg [1], with a recipe to infer the values of the strength of the Dirac- $\delta$ interaction in the ${ }^{1} S_{0}$ and ${ }^{3} S_{1}$ channels from the singlet and triplet scattering lengths, respectively. Therefore, the singlet and triplet scattering lengths make it possible to fit the renormalized strengths of the contact interactions. This effective potential should be valid for momenta well below some typical momentum scale considered in QCD, such as the $\rho$ meson mass $\left(m_{\rho} \sim 4 \mathrm{fm}^{-1}\right)$ [1], which implies in a cutoff at the momentum scale of this order or below, for the intermediate virtual propagation of the $N N$ system.

According to Weinberg's recipe, one should use naive dimensional analysis (NDA) to order terms in the potential, truncate it at a certain order, and then solve the LippmannSchwinger (LS) equation exactly. However, it has been shown that this recipe is not consistent with renormalization-group (RG) invariance, already at leading order [2-4]. The validity of Weinberg's power counting is questioned [2-4], in particular for the waves such as ${ }^{3} P_{0}$, where the singular and attractive pion tensor force requires more than two parameters even at leading order (LO).

About a decade ago, an alternative way to renormalize the nucleon-nucleon interaction, for a singular potential was proposed in Ref. [5]. In an extension of Ref. [5], the approach was proved in Ref. [6] to be RG invariant, because it satisfies the corresponding nonrelativistic Callan-Symanzik equations [7]. In this approach, no cutoff is considered in the equations and/or interactions. Instead, a subtraction point is introduced in the kernel of the LS equation [8] to reach a finite $T$ matrix. Our research group has considered singular contact interactions in the context of nuclear $[5,6,9,10]$, atomic, and general physics $[11,12]$. In such works, within specific renormalization procedures applied to few-body systems, scaling limits and correlations between low-energy observables emerge as a consequence of using singular contact (zero-range) interactions.

As the approach was proved to be invariant under RG transformations, after fitting the data, one can easily move the reference scale without affecting the physics. This flexibility in moving the reference scale is a big technical advantage of the present approach in relation to other ones. Indeed, the renormalization results for the observables should be independent of the specific scheme used. Our method was applied to the $N N$ interaction with OPEP supplemented by contact interactions $[5,6,10]$.

The significant results obtained by several authors in the implementation of the EFT program for the two-nucleon system [13-28] include a vast literature on the predictive power of the LO term (OPEP plus $\delta$ ) with a single renormalization momentum scale. Such calculation gave a sound basis for the renormalization program of EFT in the $N N$ system. In particular, the OPEP background to the $N N$ observables were carefully analyzed in Ref. [29].

Renormalization of the $N N$ interaction in chiral effective theory in LO, up to next-to-leading order (NLO), and to next-to-next-to-leading order (NNLO), in $S, P$, and $D$ waves, has been done with success in coupled and uncoupled waves [5,6,10,30-34]. References [30,31] apply the renormalization approach in coordinate space, while the works $[5,6,10,33,34]$ use subtracted-resolvent two-body techniques in momentum space. The subtracted renormalization procedure considered in Refs. [33,34] is essentially different from the one applied in Refs. $[5,6,10]$ in the way to deal with the physical inputs introduced to heal the ultraviolet divergences. In short, in the renormalization strategy of [33], one-folded subtracted equation and also a cutoff are used, implying in a window where the observables are quite independent on the cutoff. The strategy of Refs. [5,6,10] is based on the elimination of ultraviolet divergences and relies on multiple subtractions, without any additional cutoff parameter.

The multiple subtraction technique that we are going to use in the present approach demands a strategy to construct 
the driving term of the corresponding scattering equation, which is generated by consecutive subtractions in the kernel at some defined energy scale. We denote a generic order of subtractions in the kernel by $n$, such that $n=1$ stands for one subtraction. With the assumption that we have only $S$-wave LO contact interaction, one subtraction is enough. In this case the subtraction scale is set to infinity and then driven toward a given finite value of the subtraction energy by solving a nonrelativistic Callan-Symanzik (NRCS) equation $[5,6]$. The corresponding solution gives the LO driven term calculated at the reference subtraction energy where more contacts and two-pion exchange (TPE) potentials are included. We introduce each order of the potential (LO, NLO, NNLO) in the driven term of the subtracted equation when the number of subtractions are enough to make it finite. For that, we use $n=3$ in the case of NLO, and $n=4$ in NNLO. Unitarity is strictly kept. In this regard, we note that the lowest contact of the $P$-wave is introduced together with the two-pion-exchange potential (TPEP) NNLO. In the complexity of this subject, to have different approaches to tame highly divergent potentials inherent to Weinberg proposal, it is important to observe the coherence between the physical results obtained with higher order potentials and the corresponding contacts to learn subtle aspects of QCD that permeates the chiral effective expansion of the nuclear interaction. In other words, a trace of the QCD scale is expected to emerge in the fitting procedure for the nucleon-nucleon scattering data. As we are going to verify in our approach, this relic can be found in the scale associated with the subtraction point where the TPEP at NLO and NNLO are introduced in the renormalization process.

In the present work, going beyond the LO interaction and also considering $P$ - and $D$-partial waves, we show how to implement a multiple subtractive renormalization approach to obtain consistent results for the $N N$ observables. We start by summarizing the methodology used to renormalize the nucleon-nucleon interaction using a recursive subtracted approach that was previously considered in Refs. [5,6,10]. Next, we present the equations to be solved to obtain a finite $T$ matrix with NLO and NNLO interactions, taking into account partial waves up to $j=2$. The results are fitted to low-energy experimental data. As will be shown, the adjusted derivative contact terms dominate over the NLO and NNLO interactions in the $S$-wave channels for momentum higher than about $1 \mathrm{fm}^{-1}$. It is about the same, in the case of nonzero angular momentum. By including higher contact derivative terms, the potential is fitted to low-energy phase shifts up to about $200 \mathrm{MeV}$ laboratory energies.

As we have verified, the subtractive-renormalization technique considered in Refs. [5,6] is shown to be reliable also for highly singular potentials up to NNLO and $j=2$. These potentials have also been discussed in Refs. [33,34]. To show that, we present results for the nucleon-nucleon phase-shifts up to $j=2$, going beyond previous calculations within the present technique. We stress that our method cannot be naively confused with a Born approximation which, in principle, is not reliable for singular interactions. Indeed, we show that the unitarity is strictly kept along all the subtractive procedure. Moreover, it was already demonstrated in Ref. [6] that our multiple subtractive approach is fully RG invariant.
We also show how to implement the multiple subtractive renormalization method for $P$-waves with contact interactions, respecting the phase-shift threshold behavior of these waves. By considering that, we supply the details not explicitly discussed in Refs. [5,6]. Actually, $P$-wave channels were already studied in Ref. [34] with one subtraction and a sharp momentum cutoff and in Ref. [31] (and references therein), in coordinate space, even considering higher partial waves.

The number of recursive steps required to renormalize the interaction depends on how the potential diverges. For instance, the LO requires only one step, the NLO requires three steps and the NNLO requires four steps. The method has been extended for a generic number of recursive steps [6] and applied to one-pion exchange plus contact interactions with three steps [10]. Our present renormalization technique can be used to organize and implement calculations where one-pion exchange is treated nonperturbatively. Higher order terms from the chiral expansion of the nucleon-nucleon force can also be treated within this method. A further advantage of our method can be particularly appreciated in the ${ }^{1} S_{0}$ and ${ }^{3} P_{0}$ channels, in which we show the phase-shift behaviors for increasing cutoffs, exemplifying the cutoff independence as it goes to infinity. In conception, our method differs from the works presented in Refs. [33,34] and [30,31], which also dealt with simpler effective chiral expansion in the way suggested by Weinberg. In other approaches, such as the one given by Barford and Birse [22], the higher chiral orders are evaluated in distorted-wave Born approximation. However, in this case the analysis of the $\mathrm{RG}$ is quite involved. In Ref. [19], it was considered a systematic chiral procedure with a smooth cutoff applied to the potential to obtain the observables from the originally divergent scattering equations. It has also been suggested that subleading orders of the potential should not be fully iterated, but treated in (distorted-wave) perturbation theory (see, e.g., Refs. [35,36]). In our approach, we can iterate to all orders the potential and still keep the RG invariance.

This work is organized as follows. In Sec. II, we briefly describe the subtractive formalism applied to the LS equation with the RG equations. The formalism is followed by an analytical example where the RG equation is solved for the $P$-wave case with contact interactions, where two subtractions are required. In Sec. III, the renormalization formalism is applied to the NNLO potential, by considering recursive renormalization processes. The results for the $N N$ phase-shifts are presented and discussed in Sec. IV. Our conclusions are given in Sec. V.

\section{SUBTRACTED LIPPMANN-SCHWINGER EQUATION}

The scattering equation can be written with an arbitrary number of subtractions $(n)$ in the kernel $[6,10]$, which is useful when the potential has ultraviolet divergences with attractive nature; for example, $-1 / r^{m}$ with $m \geqslant 2$ (for $m=2$ the critical strength for collapsing the bound state is 1/4) and/or the potential includes contact terms (Dirac $\delta$ and its derivatives). By considering our units such that $\hbar$ and the nucleon mass $m_{N}$ 
are equal 1 , we have the energy $E$ given by $E \equiv k^{2}$. Within such units, the regularized subtracted form of the LS equation, in operatorial form, can be written as

$$
\begin{aligned}
T\left(E ;-\mu^{2}\right) & =V^{(n)}\left(E ;-\mu^{2}\right)\left[1+G^{(n)}\left(E ;-\mu^{2}\right) T\left(E ;-\mu^{2}\right)\right] \\
& =\left[1+T\left(E ;-\mu^{2}\right) G^{(n)}\left(E ;-\mu^{2}\right)\right] V^{(n)}\left(E ;-\mu^{2}\right),
\end{aligned}
$$

where, by having $H_{0}$ as the free operator, we have

$$
\begin{aligned}
G^{(n)}\left(E ;-\mu^{2}\right) \equiv & \left(\frac{\mu^{2}+E}{\mu^{2}+H_{0}}\right)^{n} G^{(0)}(E), \\
V^{(n)}\left(E ;-\mu^{2}\right)= & V^{(n-1)}\left(E ;-\mu^{2}\right)+V^{(n-1)}\left(E ;-\mu^{2}\right) \\
& \times\left(-\mu^{2}-E\right)^{n-1}\left[G^{(0)}\left(-\mu^{2}\right)\right]^{n} V^{(n)}\left(E ;-\mu^{2}\right) .
\end{aligned}
$$

We should note that $n$ is supposed to be the necessary maximum number of subtractions, with $-\mu^{2}$ the energy scaling parameter, to render finite results for the $T$ matrix. As we can verify in the above equations, the dependence on the energy starts to appear in $V^{(n)}$ for $n \geqslant 2$. The formal solution of the regularized Eq. (1), for $V^{(n)} \equiv V^{(n)}\left(E ;-\mu^{2}\right)$, is given by

$$
\begin{aligned}
T\left(E ;-\mu^{2}\right) & =\left[1-V^{(n)} G^{(n)}\left(E ;-\mu^{2}\right)\right]^{-1} V^{(n)} \\
& =V^{(n)}\left[1-G^{(n)}\left(E ;-\mu^{2}\right) V^{(n)}\right]^{-1} .
\end{aligned}
$$

By considering the operatorial expression given by Eq. (3) with $n$ subtraction, in explicit momentum-space notation, for an arbitrary single partial wave (after integrating the angular parts and with the assumption that the interaction is symmetric and not angle dependent) and by taking $V^{(n)}\left(q, p ; k^{2} ;-\mu^{2}\right)$ as the matrix element of the angular momentum projected operator, with

$$
\frac{2}{\pi} \int_{0}^{\infty} d p p^{2}|p\rangle\langle p| \equiv \mathbf{1},
$$

we obtain the partial-wave projected equation, which is given by

$$
\begin{aligned}
& V^{(n)}\left(q, p ; k^{2} ;-\mu^{2}\right) \\
& =V^{(n-1)}\left(q, p ; k^{2} ;-\mu^{2}\right)-\frac{2}{\pi} \int_{0}^{\infty} d q^{\prime} q^{\prime 2} V^{(n-1)} \\
& \quad \times\left(q, q^{\prime} ; k^{2} ;-\mu^{2}\right) \frac{\left(\mu^{2}+k^{2}\right)^{n-1}}{\left(\mu^{2}+q^{\prime 2}\right)^{n}} V^{(n)}\left(q^{\prime}, p ; k^{2} ;-\mu^{2}\right) .
\end{aligned}
$$

Therefore, as an example, we can evolve in subtractions the OPEP plus $\delta$, which is recognized to be renormalizable by fixing only one $S$ wave observable in the coupled triplet and singlet states (usually the scattering length is chosen as the input for fixing the renormalized strength of the contact). In this particular case, when only one subtraction is enough to produce a finite $T$ matrix, $V^{(1)}\left(-\mu^{2}\right)$ is replaced by the corresponding $T$ matrix at the point $E=-\mu^{2}$, such that, by defining $T\left(-\mu^{2} ;-\mu^{2}\right) \equiv T\left(-\mu^{2}\right)$, we can obtain the following subtracted equation:

$$
T\left(E ;-\mu^{2}\right)=T\left(-\mu^{2}\right)\left\{1+\left[G^{(0)}(E)-G^{(0)}\left(-\mu^{2}\right)\right] T\left(E ;-\mu^{2}\right)\right\} .
$$

For the Dirac $\delta$ potential, the matrix element in momentum space of $T\left(-\mu^{2},-\mu^{2}\right)$ is the renormalized strength.

The subtraction energy $-\mu^{2}$ in Eq. (1) can be moved without changing the resulting $T$ matrix if the driving term is evolved by solving the Callan-Symanzik equation $[6,7,10]$. The RG equation for the driving term is briefly sketched below. One example of renormalization of a $P$-wave case, with our method, is also discussed for illustration. On the calculations of shallow $P$-wave states, with EFT theory applied for halo nuclei, see Ref. [37].

In addition, we remark on a general feature of the subtraction procedure applied to attractive and repulsive power-law potentials in configuration space $\left(r^{-m}\right.$ with $\left.m \geqslant 2\right)$. In the repulsive case the subtraction is not trivially required to render finite the $T$ matrix, as the scattering wave function is damped in the classically forbidden region. In contrast, in the attractive case the ultraviolet divergence is actually met dynamically and may collapse the state. That is the case that a regularization has to be performed at the short range. In our method we deal with this problem, by using enough subtractions to make the $T$-matrix finite. Attractive and repulsive terms that we are discussing now appear as the chiral expansion is performed, as also discussed thoroughly by Valderrama and Arriola in their renormalization scheme in configuration space for calculation of the $N N$ phase shifts [30]. Instead, in our approach, subtractions are introduced to include the contacts, at the expense of finding a convenient subtraction point with the meaning of a reference low-energy scale. As we show below, indeed, the fitting of partial waves phase shifts up to $j=2$ gives a reference subtraction energy between -50 and $-100 \mathrm{MeV}$. At this scale the NLO and NNLO TPEP potentials are identified as contributions to the driving terms as we are going to explain in detail in Sec. III.

Note that the reference subtraction point is, in principle, arbitrary and can be moved by evolving the potential through the $\mathrm{RG}$ flow equation. Once the fitting to the data is done, the reference scale can be changed arbitrarily as long as the driving term (starting potential) is evolved through the NRCS flow equation [7]. In fact, this implies that the RG invariance cannot be separated from the form of the evolved interaction. Next, we briefly discuss the RG equations, followed by an analytical example.

\section{A. Renormalization group equations}

The nucleon-nucleon observables are invariant under the change of the arbitrary subtraction point; therefore, one can start at any convenient energy scale $-\mu^{2}$. However, the form of the driving term and its coefficients, which define the scattering amplitude, are tied to the prescription used to define the renormalized theory. The key point of the RG method is to change this prescription without altering the predictions of the theory [38].

The invariance of the $T$ matrix under changes of renormalization prescriptions, that is,

$$
\frac{\partial}{\partial \mu^{2}} T\left(E ;-\mu^{2}\right)=0,
$$


imposes a definite rule to modify $V^{(n)}$ that appears in a form of a NRCS equation [6,7]:

$$
\begin{aligned}
& \frac{\partial V^{(n)}\left(E ;-\mu^{2}\right)}{\partial \mu^{2}} \\
& =-V^{(n)}\left(E ;-\mu^{2}\right) \frac{\partial G^{(n)}\left(E ;-\mu^{2}\right)}{\partial \mu^{2}} V^{(n)}\left(E ;-\mu^{2}\right) \\
& =n V^{(n)}\left(E ;-\mu^{2}\right) \frac{\left(E+\mu^{2}\right)^{n-1}}{\left(H_{0}+\mu^{2}\right)^{n+1}} V^{(n)}\left(E ;-\mu^{2}\right),
\end{aligned}
$$

which is derived from the invariance of $T\left(E ;-\mu^{2}\right)$, given by Eq. (4), with respect to the renormalization parameter $\mu^{2}$. The demonstration of the above can easily be done by considering both expressions that appear in Eq. (4). Equation (8) substantiate the invariance of the renormalized $T$ matrix under dislocation of the subtraction point. Then we observe that there is a nontrivial dependence on the subtraction point appearing in the driving term of the subtracted scattering equation, although the physical results of the model are kept unchanged. Thus, from now on, we drop the explicit dependence on $\mu^{2}$ in the $T$ matrix by writing $T\left(E ;-\mu^{2}\right) \equiv T(E)$.

The solution of Eq. (8) implies in a complicated evolution of $V^{(n)}$ as $\mu$ changes. Not only the strengths of the interactions would change, but also the form of the driving term. The ultraviolet behavior of the driving term is not changed by the evolution in $\mu$. The evolution should not be truncated as $\mu$ is varied to keep the $T$ matrix invariant. At different $\mu$ the potential $V^{(n)}\left(-\mu^{2} ; E\right)$ has a complicated form from the solution of NRCS equation. Similarly, the evolution of RG equations as introduced by Bogner, Kuo, and Schwenk [39] for the $N N$ scattering does not truncate on certain operators as the cutoff is varied to keep the observables unchanged.

\section{B. Example: Subtracted $\boldsymbol{P}$-wave equations with contact interactions}

We illustrate the use of our multiple subtraction renormalization method by discussing the case of a one-channel $P$-wave problem with a derivative contact interaction $V\left(p, p^{\prime}\right)=$ $\lambda_{1} p p^{\prime}$. The $P$-wave LS equation with a momentum cutoff $\Lambda$ is easily solvable, with the corresponding $T$ matrix given by

$$
T\left(p, p^{\prime} ; k^{2}\right)=p \cdot p^{\prime}\left(\frac{1}{\lambda_{1}}-\frac{2}{\pi} \int_{0}^{\Lambda} d q q^{4} \frac{1}{k^{2}-q^{2}+\mathrm{i} \epsilon}\right)^{-1} .
$$

The coefficient $\lambda_{1}$ can be fixed by the scattering volume $\alpha$, which gives

$$
\frac{1}{\lambda_{1}}=-\frac{2}{3 \pi} \Lambda^{3}+\frac{1}{\alpha}
$$

By replacing $\lambda_{1}$ in Eq. (10), we have

$$
T\left(p, p^{\prime} ; k^{2}\right)=p \cdot p^{\prime}\left(\frac{1}{\alpha}-\frac{2}{\pi} k^{2} \int_{0}^{\Lambda} d q q^{2} \frac{1}{k^{2}-q^{2}+\mathrm{i} \epsilon}\right)^{-1},
$$

which shows that this procedure is not enough to eliminate the cutoff dependence of Eq. (10). With this simple example, we can see that a renormalization procedure using only one subtraction, for a $P$-wave problem, is expected to be cutoff dependent, requiring a second subtraction.

The fit of only the scattering volume is equivalent in our method to using one subtraction in the LS equation. One should note that for one subtraction $n=1$, the dependence on the cutoff is linear. Even in this simple example, the RG evolution of the recursive driving term is not straightforward. However, it is important to stress that one more parameter is required to renormalize Eq. (12). Therefore, in agreement with Refs. $[37,40]$, to renormalize the $P$-wave contact interaction we need two inputs: the scattering volume and the next term in the generalized effective range expansion for the $k^{3} \cot \delta$.

Within our method, with a scaling momentum parameter $\mu$, instead of the cutoff $\Lambda$, the ultraviolet divergence in the scattering equation from the potential $V\left(p, p^{\prime}\right)=\lambda_{1} p p^{\prime}$ requires two subtractions $(n=2)$ to render finite the integral appearing in Eq. (10). The corresponding result, where we can take the limit $\Lambda \rightarrow \infty$, is given by

$$
\begin{aligned}
\frac{T\left(p, p^{\prime} ; k^{2}\right)}{p p^{\prime}}= & \left(\frac{1}{\lambda_{1}\left(k^{2}, \mu^{2}\right)}\right. \\
& \left.-\frac{2}{\pi} \int_{0}^{\infty} d q \frac{q^{4}\left(k^{2}+\mu^{2}\right)^{2}}{\left(\mu^{2}+q^{2}\right)^{2}\left(k^{2}-q^{2}+\mathrm{i} \epsilon\right)}\right)^{-1},
\end{aligned}
$$

where the parameter $\lambda_{1}$ is now finite and can be fitted by the scattering volume. However, a dependence on $\mu$ is intrinsic to this method, unless the driving term is evolved through a NRCS equation $[6,10]$.

Just to give an impression on how the RG equation works for the $P$-wave example, we introduce the renormalized driving term $V\left(p, p^{\prime}\right) \equiv V\left(p, p^{\prime} ; k^{2}\right)=\lambda_{1}\left(k^{2}, \mu^{2}\right) p p^{\prime}$ in Eq. (9). After projection in the $P$-wave, we have

$$
\begin{aligned}
\frac{\partial \lambda_{1}\left(k^{2}, \mu^{2}\right)}{\partial \mu^{2}} & =2\left[\lambda_{1}\left(k^{2}, \mu^{2}\right)\right]^{2} \frac{2}{\pi} \int_{0}^{\infty} d q q^{4} \frac{\left(k^{2}+\mu^{2}\right)}{\left(q^{2}+\mu^{2}\right)^{3}} \\
& =\frac{3}{4} \frac{\left(k^{2}+\mu^{2}\right)}{\mu}\left[\lambda_{1}\left(k^{2}, \mu^{2}\right)\right]^{2}
\end{aligned}
$$

Therefore, the solution of the RG equation gives

$$
\frac{1}{\lambda_{1}\left(k^{2}, \mu^{2}\right)}=\frac{1}{\lambda_{1}\left(k^{2}, \mu_{0}^{2}\right)}-\frac{3}{2} k^{2}\left(\mu-\mu_{0}\right)-\frac{1}{2}\left(\mu^{3}-\mu_{0}^{3}\right) .
$$

Note that, even if at the reference subtraction energy $-\mu_{0}^{2}$ the renormalized strength is chosen to be independent of $k^{2}$, the evolution introduces a $k^{2}$ dependence, and implicitly the need of two-subtractions to render finite the $T$ matrix for the $P$-wave. If one fixes the reference scale at zero energy, as suggested in Ref. [34], the renormalized strength is the scattering volume assuming independence with $k^{2}$ at this reference energy. In our case, after solving the RG equation, the form of the evolved potential is simply the renormalized $\lambda_{1}$ from Eq. (15) times $p p^{\prime}$. Solving Eq. (13) with the 
renormalized coupling from Eq. (15), we get

$$
k^{3} \cot \delta=-\frac{1}{\lambda_{1}\left(k^{2}, \mu^{2}\right)}-\frac{\mu}{2}\left(3 k^{2}+\mu^{2}\right) .
$$

The evolution of $\lambda_{1}$ according to Eq. (15) shows that it should be a linear function of $k^{2}$, which demands two constants. Indeed, if we rewrite Eq. (16) taking into account the constant plus the $k^{2}$ term from $\lambda_{1}$ only two independent quantities appear, that is, a scattering volume and an effective momentum. In fact, the form we obtain for $k^{3} \cot \delta$ is similar to the one obtained in Ref. [37].

\section{RENORMALIZATION OF THE NNLO POTENTIAL WITH $n=4$}

Once we have established the recursive procedure to renormalize the $N N$ interaction, we need a potential in momentum space. For the NNLO chiral potential, we adopt a momentum-space form as explicitly given by Epelbaum [19].

Even though our method is powerful enough to renormalize the full TPE potential, we consider the version with the spectral representation regularization such that the comparison with results obtained by other calculations can be more straight.

For the sake of completeness and the reader's convenience, we repeat here the analytical expressions for the chiral NNLO momentum space potential. The LO interaction is given by the one-pion exchange (OPE) plus a contact interaction. The strength of the isospin conserving contact terms and their derivatives depends on the orbital-spin channel of the total angular momentum $\mathrm{J}$, considering also angular momentum mixing. We simplify our notation for the contact terms so that the total angular momentum and isospin dependence are not shown explicitly in the expressions for the potential. The corresponding $S$-wave projected matrix elements of the interaction is given by

$$
V_{\mathrm{LO}}\left(p, p^{\prime}\right)=V_{\mathrm{OPE}}\left(p, p^{\prime}\right)+\lambda_{0},
$$

where the unprojected OPE potential, for $\vec{q} \equiv \vec{p}-\vec{p}^{\prime}$, is given by

$$
V_{\mathrm{OPE}}\left(\vec{p}, \vec{p}^{\prime}\right)=\frac{-1}{(2 \pi)^{3}}\left(\frac{g_{A}}{2 f_{\pi}}\right)^{2} \boldsymbol{\tau}_{1} \cdot \boldsymbol{\tau}_{2} \frac{\left(\vec{\sigma}_{1} \cdot \vec{q}\right)\left(\vec{\sigma}_{2} \cdot \vec{q}\right)}{q^{2}+M_{\pi}^{2}},
$$

At NLO, we have some TPE diagrams plus derivative contact interactions. After partial wave projection, it is given by

$$
\begin{aligned}
V_{\mathrm{NLO}}\left(p, p^{\prime}\right)= & V_{\mathrm{TPE}}^{\mathrm{NLO}}\left(p, p^{\prime}\right)+\lambda_{1}\left(p p^{\prime}\right) \delta_{L, 1} \delta_{L^{\prime}, 1} \\
& +\left(\lambda_{2}\left(p^{2}+p^{\prime 2}\right)+\lambda_{3}\left(p^{2} p^{\prime 2}\right)\right) \delta_{L, 0} \delta_{L^{\prime}, 0} \\
& +\lambda_{4}\left(p^{2} \delta_{L, 2} \delta_{L^{\prime}, 0}+p^{\prime 2} \delta_{L^{\prime}, 2} \delta_{L, 0}\right),
\end{aligned}
$$

where the unprojected NLO TPE potential is

$$
\begin{aligned}
V_{\mathrm{TPE}}^{\mathrm{NLO}}\left(\vec{p}, \vec{p}^{\prime}\right)= & -\left(\frac{\boldsymbol{\tau}_{1} \cdot \boldsymbol{\tau}_{2}}{384 \pi^{2} f_{\pi}^{4}}\right) \frac{L(q)}{(2 \pi)^{3}}\left\{4 M_{\pi}^{2}\left(5 g_{A}^{4}-4 g_{A}^{2}-1\right)\right. \\
& \left.+q^{2}\left(23 g_{A}^{4}-10 g_{A}^{2}-1\right)+\frac{48 g_{A}^{4} M_{\pi}^{4}}{4 M_{\pi}^{2}+q^{2}}\right\} \\
& -\left(\frac{3 g_{A}^{4}}{64 \pi^{2} f_{\pi}^{4}}\right) L(q)\left\{\left(\vec{\sigma}_{1} \cdot \vec{q}\right)\left(\vec{\sigma}_{2} \cdot \vec{q}\right)\right. \\
& \left.-q^{2} \vec{\sigma}_{1} \cdot \vec{\sigma}_{2}\right\} .
\end{aligned}
$$

The term proportional to $\lambda_{1}$ contributes only in the $P$ waves, the terms proportional to $\lambda_{2}$ and $\lambda_{3}$ appear only in the $S$ waves, and the the term proportional to $\lambda_{4}$ enters only in the coupled channels with $j=1$. The $\lambda_{3}$ term $\left(p^{2} p^{\prime 2}\right)$ actually appears at $\mathrm{N}^{3} \mathrm{LO}$ in the Weinberg's power counting, but we promoted it to NLO to improve the $S$ waves fit. It could have been promoted to NNLO, but because the iteration of the NLO $\lambda_{2}$ term $\left(p^{2}+p^{\prime 2}\right)$ leads to $p^{2} p^{\prime 2}$ terms, we included it at NLO so that all the $p^{2} p^{\prime 2}$ terms combine at once. This has also been done in Ref. [10].

Finally, at NNLO, we have other TPE diagrams, with the corresponding unprojected potential given by

$$
\begin{aligned}
V_{\mathrm{NNLO}}\left(\vec{p}, \vec{p}^{\prime}\right) \equiv & V_{\mathrm{TPE}}^{\mathrm{NNLO}}\left(\vec{p}, \vec{p}^{\prime}\right)=\frac{1}{(2 \pi)^{3}}\left(\frac{3 g_{A}^{2}}{16 \pi f_{\pi}^{4}}\right)\left\{\frac{g_{A}^{2} M_{\pi}^{5}}{16 m\left(4 M_{\pi}^{2}+q^{2}\right)}-\left[2 M_{\pi}^{2}\left(2 c_{1}-c_{3}\right)-q^{2}\left(c_{3}+\frac{3 g_{A}^{2}}{16 m}\right)\right]\left(2 M_{\pi}^{2}+q^{2}\right) A(q)\right\} \\
& -\frac{g_{A}^{2}}{128 \pi m f_{\pi}^{4}}\left(\boldsymbol{\tau}_{1} \cdot \boldsymbol{\tau}_{2}\right)\left\{-\frac{3 g_{A}^{2} M_{\pi}^{5}}{4 M_{\pi}^{2}+q^{2}}+\left[4 M_{\pi}^{2}+2 q^{2}-g_{A}^{2}\left(4 M_{\pi}^{2}+3 q^{2}\right)\right]\left(2 M_{\pi}^{2}+q^{2}\right) A(q)\right\} \\
& +\frac{9 g_{A}^{4}}{512 \pi m f_{\pi}^{4}}\left[\left(\vec{\sigma}_{1} \cdot \vec{q}\right)\left(\vec{\sigma}_{2} \cdot \vec{q}\right)-q^{2}\left(\vec{\sigma}_{1} \cdot \vec{\sigma}_{2}\right)\right]\left(2 M_{\pi}^{2}+q^{2}\right) A(q)-\frac{g_{A}^{2}}{32 \pi f_{\pi}^{4}}\left(\boldsymbol{\tau}_{1} \cdot \boldsymbol{\tau}_{2}\right) \\
& \times\left[\left(\vec{\sigma}_{1} \cdot \vec{q}\right)\left(\vec{\sigma}_{2} \cdot \vec{q}\right)-q^{2}\left(\vec{\sigma}_{1} \cdot \vec{\sigma}_{2}\right)\right]\left\{\left(c_{4}+\frac{1}{4 m}\right)\left(4 M_{\pi}^{2}+q^{2}\right)-\frac{g_{A}^{2}}{8 m}\left(10 M_{\pi}^{2}+3 q^{2}\right)\right\} A(q)-\frac{3 g_{A}^{4}}{64 \pi m f_{\pi}^{4}} i\left(\vec{\sigma}_{1}+\vec{\sigma}_{2}\right) \\
& \cdot\left(\vec{p}^{\prime} \times \vec{p}\right)\left(2 M_{\pi}^{2}+q^{2}\right) A(q)-\frac{g_{A}^{2}\left(1-g_{A}^{2}\right)}{64 \pi m f_{\pi}^{4}}\left(\boldsymbol{\tau}_{1} \cdot \boldsymbol{\tau}_{2}\right) i\left(\vec{\sigma}_{1}+\vec{\sigma}_{2}\right) \cdot\left(\vec{p}^{\prime} \times \vec{p}\right)\left(4 M_{\pi}^{2}+q^{2}\right) A(q),
\end{aligned}
$$

where the loop integrals $L(q)$ and $A(q)$ are given by

$$
\begin{gathered}
L(q)=\frac{1}{q} \sqrt{4 M_{\pi}^{2}+q^{2}} \ln \frac{\sqrt{4 M_{\pi}^{2}+q^{2}}+q}{2 M_{\pi}} \approx \theta\left(\tilde{\Lambda}-2 M_{\pi}\right) \frac{\sqrt{4 M_{\pi}^{2}+q^{2}}}{2 q} \ln \frac{\left(\tilde{\Lambda} \sqrt{4 M_{\pi}^{2}+q^{2}}+q \sqrt{\tilde{\Lambda}^{2}-4 M_{\pi}^{2}}\right)^{2}}{4 M_{\pi}^{2}\left(\tilde{\Lambda}^{2}-q^{2}\right)}, \\
A(q)=\frac{1}{2 q} \arctan \frac{q}{2 M_{\pi}}, \approx \theta\left(\tilde{\Lambda}-2 M_{\pi}\right) \frac{1}{2 q} \arctan \frac{q\left(\tilde{\Lambda}-2 M_{\pi}\right)}{q^{2}+2 M_{\pi} \tilde{\Lambda}} . \\
064005-5
\end{gathered}
$$


In the above expressions, $\tilde{\Lambda}$ is a spectral regularization scale defined in Ref. [19] for the two-pion exchange, to improve the convergence of the chiral expansion. As suggested $\tilde{\Lambda} \approx$ four-pion mass, we set $\tilde{\Lambda}=600 \mathrm{MeV}$.

\section{A. Evolving the potentials through the recursive renormalization process}

We start by calculating $V^{(1)}\left(-\mu^{2}\right)$ from the LO interaction $V_{\mathrm{LO}}$, by solving the Callan-Symanzik equation (8) for $n=1$ starting at a negative infinite $\bar{\mu}^{2}$ up to a finite $\mu^{2}$. The integral form of RG equation (8) for $n=1$, after partial wave decomposition, is

$$
\begin{aligned}
& V^{(1)}\left(p, p^{\prime} ;-\mu^{2}\right) \\
& =V_{\mathrm{LO}}\left(p, p^{\prime} ;-\bar{\mu}^{2}\right)+\frac{2}{\pi} \int_{0}^{\infty} d q q^{2} \\
& \quad \times \frac{V_{\mathrm{LO}}\left(p, q ;-\bar{\mu}^{2}\right)\left(\mu^{2}-\bar{\mu}^{2}\right)}{\left(\bar{\mu}^{2}+q^{2}\right)\left(\mu^{2}+q^{2}\right)} V^{(1)}\left(q, p^{\prime} ;-\mu^{2}\right),
\end{aligned}
$$

which brings the LO interaction to a scale $-\mu^{2}$ from its infinitely large fixed-point $-\bar{\mu}^{2}[5,10,13,22]$. This generates an interaction for the one subtracted scattering equation, which gives the same observables as the LO interaction when $\mu \gg \Lambda_{\mathrm{QCD}}$. Now we obtain $V^{(2)}\left(p, p^{\prime} ; k^{2} ;-\mu^{2}\right)$ from $V^{(1)}\left(p, p^{\prime} ;-\mu^{2}\right)$ :

$$
\begin{aligned}
& V^{(2)}\left(p, p^{\prime} ; k^{2} ;-\mu^{2}\right) \\
&= V^{(1)}\left(p, p^{\prime} ;-\mu^{2}\right)-\frac{2}{\pi} \int_{0}^{\infty} d q q^{2} \\
& \times \frac{V^{(1)}\left(p, q ;-\mu^{2}\right)\left(\mu^{2}+k^{2}\right)}{\left(\mu^{2}+q^{2}\right)^{2}} V^{(2)}\left(q, p^{\prime} ; k^{2} ;-\mu^{2}\right) .
\end{aligned}
$$

At the third step we evolve from $V^{(2)}$ and introduce the NLO terms

$$
\begin{aligned}
\bar{V}^{(3)}\left(p, p^{\prime} ; k^{2} ;-\mu^{2}\right)= & V^{(2)}\left(p, p^{\prime} ; k^{2} ;-\mu^{2}\right)-\frac{2}{\pi} \int_{0}^{\infty} \\
& \times d q q^{2} \frac{V^{(2)}\left(p, q ; k^{2} ;-\mu^{2}\right)\left(\mu^{2}+k^{2}\right)^{2}}{\left(\mu^{2}+q^{2}\right)^{3}} \\
& \times \bar{V}^{(3)}\left(q, p^{\prime} ; k^{2} ;-\mu^{2}\right), \\
V^{(3)}\left(p, p^{\prime} ; k^{2} ;-\mu^{2}\right)= & V_{\mathrm{NLO}}\left(p, p^{\prime} ;-\mu^{2}\right) \\
& +\bar{V}^{(3)}\left(p, p^{\prime} ; k^{2} ;-\mu^{2}\right) .
\end{aligned}
$$

At the fourth step, the higher order we consider here, we evolve from $V^{(3)}$ and add the NNLO two-pion exchange:

$$
\begin{aligned}
\bar{V}^{(4)}\left(p, p^{\prime} ; k^{2} ;-\mu^{2}\right)= & V^{(3)}\left(p, p^{\prime} ; k^{2} ;-\mu^{2}\right) \\
& -\frac{2}{\pi} \int_{0}^{\infty} d q q^{2} V^{(3)}\left(p, q ; k^{2} ;-\mu^{2}\right) \\
& \times \frac{\left(\mu^{2}+k^{2}\right)^{3}}{\left(\mu^{2}+q^{2}\right)^{4}} \bar{V}^{(4)}\left(q, p^{\prime} ; k^{2} ;-\mu^{2}\right) \\
V^{(4)}\left(p, p^{\prime} ; k^{2} ;-\mu^{2}\right)= & V_{\mathrm{NNLO}}\left(p, p^{\prime} ;-\mu^{2}\right) \\
& +\bar{V}^{(4)}\left(p, p^{\prime} ; k^{2} ;-\mu^{2}\right) .
\end{aligned}
$$

TABLE I. Strengths of the LO contact interactions, which reproduce the scattering lengths for the $S$ waves. The values of $\lambda_{0}^{1} S_{0}$ and $\lambda_{0}^{3} S_{1}$, in units of $\mathrm{fm}$, are given at the energy scale $-\bar{\mu}^{2}$, with $\bar{\mu}=30 \mathrm{fm}^{-1}\left(\bar{\mu}^{2}=41.47 \times 900 \mathrm{MeV}\right)$.

\begin{tabular}{lcc}
\hline \hline Strengths & ${ }^{1} S_{0}$ & ${ }^{3} S_{1}$ \\
\hline$\lambda_{0}(\mathrm{fm})$ & -0.0203 & -0.24142 \\
\hline \hline
\end{tabular}

With the above, the half-on-shell $T$ matrix with four subtractions is a solution of

$$
\begin{aligned}
T\left(p, k ; k^{2}\right)= & V^{(4)}\left(p, k ; k^{2} ;-\mu^{2}\right)+\frac{2}{\pi} \int_{0}^{\infty} d p^{\prime} p^{\prime 2} V^{(4)} \\
& \times\left(p, p^{\prime} ; k^{2} ;-\mu^{2}\right)\left(\frac{\mu^{2}+k^{2}}{\mu^{2}+p^{\prime 2}}\right)^{4} \frac{1}{k^{2}-p^{\prime 2}+\mathrm{i} \epsilon} \\
& \times T\left(p^{\prime}, k ; k^{2}\right) .
\end{aligned}
$$

Note that in the above equation the term given by $\left[\left(\mu^{2}+\right.\right.$ $\left.\left.k^{2}\right) /\left(\mu^{2}+p^{\prime 2}\right)\right]^{4}$ works effectively as a regulator, canceling the singularity presented in the starting interaction. The driving term is generated by consecutive subtractions in the kernel at some defined energy scale $\mu^{2}$, with the advantage that such scale can be moved freely as long as $V^{(4)}$ satisfies the RG equation, given by Eq. (8), that guarantees that the scattering amplitude is unaltered.

It is important to explain the strategy used to insert the potential in the recursive to obtain the driving term $V^{(4)}$ that enters in Eq. (27). We include each order of the interaction (LO, NLO, NNLO) in the step where there is enough subtractions to renormalize it. Consequently, we insert the LO potential in the first subtraction, the NLO potential in the third subtraction, and so on. Tables I to IV list the parameters for the potentials used in this work.

Before closing this section, we discuss briefly our scheme and the one used in Ref. [34], where $S$ waves have been considered with more than one subtraction. In that work, the successive subtractions are performed in a rather different form compared to our approach. They call it a mixed scheme. The main difference is: To handle the momentum-dependent contact interaction $p^{2}+p^{\prime 2}$, the cutoff also plays an important role in Ref. [34], because according to our method this interaction actually requires three subtractions. In our case, we can approach any kind of interaction as long as we perform

TABLE II. Strengths of the contact interactions for the fits with the LO potential plus the NLO contact interactions. The values of $\lambda_{0}^{1} S_{0}$ and $\lambda_{0}^{3} S_{1}$ are given at the same energy scale as in Table $\mathrm{I}\left(-\bar{\mu}^{2}=\right.$ $-41.47 \times 900 \mathrm{MeV})$; with $\lambda_{2}^{1} S_{0}$ and $\lambda_{3}^{1} S_{0}$ at $-\mu^{2}=-50 \mathrm{MeV}$. The other strengths are given at $-\mu^{2}=-100 \mathrm{MeV}$.

\begin{tabular}{lccccccc}
\hline \hline Strengths & ${ }^{1} S_{0}$ & ${ }^{3} P_{0}$ & ${ }^{3} S_{1}$ & ${ }^{1} P_{1}$ & ${ }^{3} P_{1}$ & ${ }^{3} P_{2}$ & $\epsilon_{1}$ \\
\hline$\lambda_{0}(\mathrm{fm})$ & -0.0165 & - & -0.2480 & - & - & - & - \\
$\lambda_{1}\left(\mathrm{fm}^{3}\right)$ & - & 0.25 & - & 0.04 & 0.007 & -0.07 & - \\
$\lambda_{2}\left(\mathrm{fm}^{3}\right)$ & 2.2660 & - & 0.1 & - & - & - & - \\
$\lambda_{3}\left(\mathrm{fm}^{5}\right)$ & 2.0047 & - & - & - & - & - & - \\
$\lambda_{4}\left(\mathrm{fm}^{3}\right)$ & - & - & - & - & - & - & 0.001 \\
\hline \hline
\end{tabular}


TABLE III. Strengths of the contact interactions for the fits with the full NLO potential. The values of the $\lambda$ 's are given for $\bar{\mu}^{2}$ and $\mu^{2}$ as in Table II.

\begin{tabular}{lccccccc}
\hline \hline Strengths & ${ }^{1} S_{0}$ & ${ }^{3} P_{0}$ & ${ }^{3} S_{1}$ & ${ }^{1} P_{1}$ & ${ }^{3} P_{1}$ & ${ }^{3} P_{2}$ & $\epsilon_{1}$ \\
\hline$\lambda_{0}$ & -0.0190 & - & -0.1602 & - & - & - & - \\
$\lambda_{1}\left(\mathrm{fm}^{3}\right)$ & - & 0.37 & - & 0.063 & -0.078 & -0.04 & - \\
$\lambda_{2}\left(\mathrm{fm}^{3}\right)$ & 2.2660 & - & 0.1 & - & - & - & - \\
$\lambda_{3}\left(\mathrm{fm}^{5}\right)$ & 2.0047 & - & - & - & - & - & - \\
$\lambda_{4}\left(\mathrm{fm}^{3}\right)$ & - & - & - & - & - & - & 0.17 \\
\hline \hline
\end{tabular}

enough subtractions. The cutoff has no physical relevance because the multiple subtractions allow us to sum up to arbitrarily large momentum values.

\section{B. Half-on-shell amplitudes for the recursive renormalization process}

Now, let us consider the calculation of the half-on shell matrices $V^{(n)}(q, k)$ for $n=1$ to 4 , from the solution of Eqs. (23)-(26) and the half-on-shell matrix elements of $T\left(q, k ; k^{2}\right)$ solution of Eq. (27). Before starting our discussion, a side remark is worthwhile. As we have seen before, from the above equations, the recursive driving terms obey integral equations with kernels defined by subtracted free Green's functions multiplied by recursive driving terms one order below. From the subtracted method itself the lowest order recursive driving term satisfies an equation which is energy independent. In the following orders the integral equations for the driving term depend on $k^{2}$, and the subtracted Green's function is negative, which brings a curious effect on the respective solutions of Eq. (6). The corresponding homogeneous equation, in the case of attractive recursive interaction, suffers an enhancement by increasing $k^{2}$, owing to the factor $\left(\mu^{2}+k^{2}\right)$ inside the kernel, which may allow it to have a solution. When this happens, an unphysical pole will occur in the recursive interaction of the integral equation. This can be realized even with a regular attractive potential if multiple subtractions are used to compute the scattering amplitude. This unphysical pole is completely washed out in the solution of the $n$-subtracted LS equation given below.

We can observe the half-on-shell recursive driving terms evolving through the four subtractions from $V^{(1)}(q, k)$ up to $t(q, k)$ for some values of the on-shell momentum $k$ in Figs. 1 to 4 for the ${ }^{1} S_{0}$ and ${ }^{3} S_{1}$ channels. In Figs. 1 and 3 we have the

TABLE IV. Strengths of the contact interactions for the fits with the NNLO potential. The values of the $\lambda$ 's are given for $\bar{\mu}^{2}$ and $\mu^{2}$ as in Table II.

\begin{tabular}{lccccccc}
\hline \hline Strengths & ${ }^{1} S_{0}$ & ${ }^{3} P_{0}$ & ${ }^{3} S_{1}$ & ${ }^{1} P_{1}$ & ${ }^{3} P_{1}$ & ${ }^{3} P_{2}$ & $\epsilon_{1}$ \\
\hline$\lambda_{0}(\mathrm{fm})$ & -0.0189 & - & -0.1217 & - & - & - & - \\
$\lambda_{1}\left(\mathrm{fm}^{3}\right)$ & - & 0.303 & - & 0.066 & -0.19 & -0.1 & - \\
$\lambda_{2}\left(\mathrm{fm}^{3}\right)$ & 2.2660 & - & 0.1 & - & - & - & - \\
$\lambda_{3}\left(\mathrm{fm}^{5}\right)$ & 2.0047 & - & - & - & - & - & - \\
$\lambda_{4}\left(\mathrm{fm}^{3}\right)$ & - & - & - & - & - & - & 0.17 \\
\hline \hline
\end{tabular}
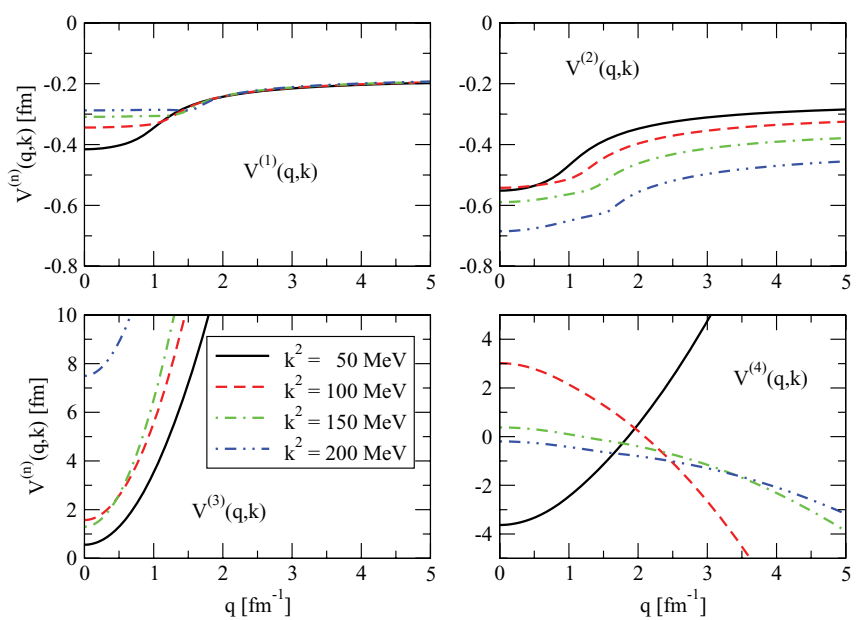

FIG. 1. (Color online) Half-on-shell recursive driving terms $V^{(n)}\left(q, k ; k^{2}\right)$ for the ${ }^{1} S_{0}$ channel, corresponding to the energies $E=(\hbar k)^{2} / m_{N}=50,100,150$, and $200 \mathrm{MeV}$ (with units such that $\hbar=1$ and $m_{N}=1$ ). The lines' identification, shown inside the bottom-left panel, is given for all four panels. The values of the strengths, as well as the subtraction energy scales (not explicitly shown here), are given in Tables I-IV.

driving terms and half-shell $T$-matrix elements, respectively, for the ${ }^{1} S_{0}$ channel. Correspondingly, we have the results for the ${ }^{3} S_{1}$ channel in Figs. 2 and 4 . These figures exhibit us an interesting finding owing to Redish and Stickbauer [41]. They observe that distinct half-on-shell potentials may lead to similar half-on-shell $T$ matrixes. In other words, given different $V(q, k)$ which fit the same on-shell observables their corresponding half-on-shell $T$ matrix should be quite equivalent despite the $V(q, k)$ discrepancies. That is why we observe a smooth behavior of the scattering amplitude with energy in Fig. 2 while the recursive driving terms, in particular $V^{(4)}$, vary considerably, as can be seen in the bottom right panel of Fig. 1.

The set of integral equations for the subtracted driving terms Eqs. (23)-(26) given above also deserves further comment to

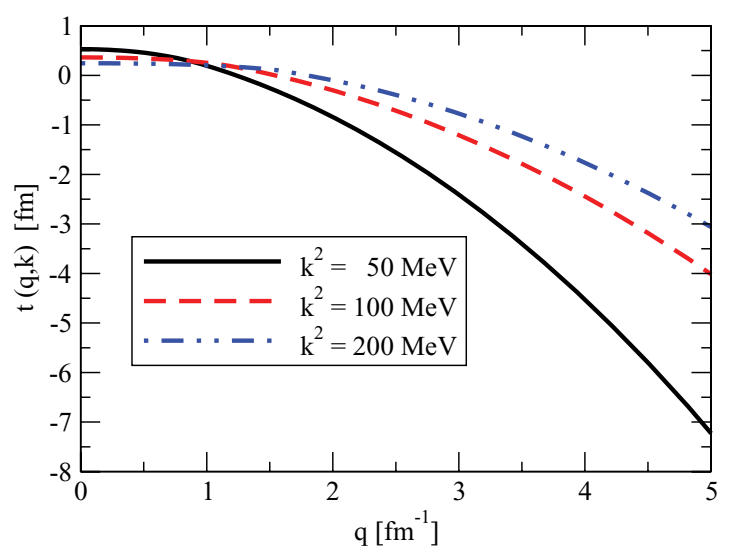

FIG. 2. (Color online) Half-on-shell $T$-matrix elements for the ${ }^{1} S_{0}$ channel, for the energies $k^{2}=50,100$, and $200 \mathrm{MeV}$. As in Fig. 1, the values of the strengths, as well as the subtraction energy scales (not explicitly shown here), are given in Tables I-IV. 

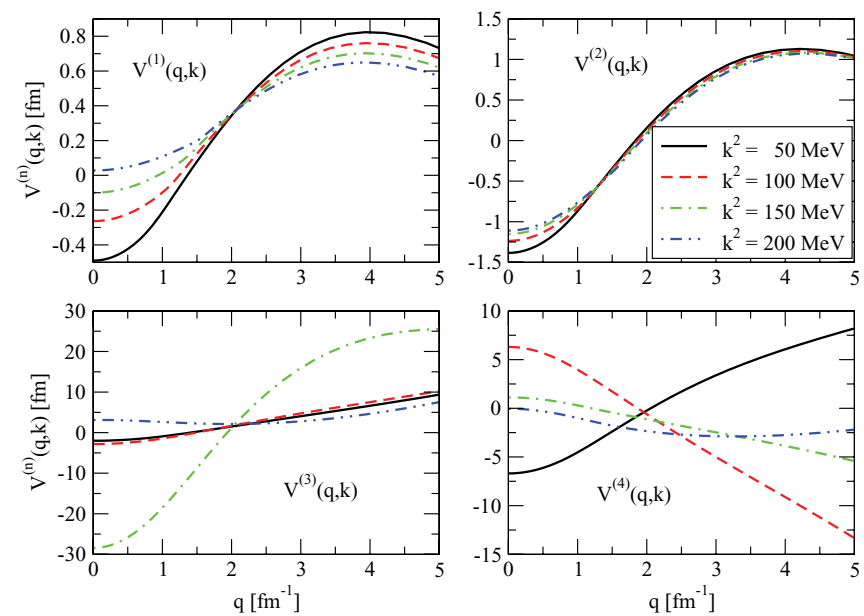

FIG. 3. (Color online) Half-on-shell recursive driving terms $V^{(n)}\left(q, k ; k^{2}\right)$ for the ${ }^{3} S_{1}$ channel, corresponding to the energies $k^{2}=$ $50,100,150$, and $200 \mathrm{MeV}$, as given inside the top-right panel. As in Fig. 1, the values of the strengths, as well as the subtraction energy scales (not explicitly shown here), are given in Tables I-IV.

remove the naive misconception that the approach given in [10] "involves invoking the Born approximation and consequently unreliable for the higher singular potentials" [34]. As one should observe, such an approach relies on the definition of a driving term $V^{(n)}$ at each subtraction order. Of course, such term is the transition matrix at the subtraction point. The driving term should be evolved dynamically by the renormalization procedure to the next order, where higher divergent interactions are added, if necessary. If a contact interaction is included, its strength is the renormalized one at that point. Such a procedure continues up to the number of subtractions required to render finite the corresponding scattering equation. Let us emphasize that, differently from the usual Born approximation (where the dynamics does not evolve the potential), this renormalization procedure relies on a recursive evolution of the driving term, from one order to the next one. Moreover, it produces a unitary $S$ matrix.

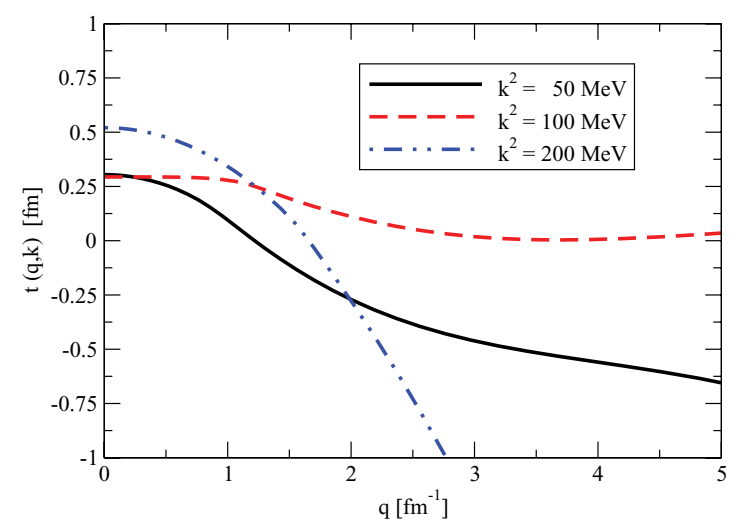

FIG. 4. (Color online) Half-on-shell $T$-matrix elements for the ${ }^{3} S_{1}$ channel, for the energies $k^{2}=50,100,150$, and $200 \mathrm{MeV}$. As in Fig. 1, the values of the strengths, as well as the subtraction energy scales (not explicitly shown here), are given in Tables I-IV.
The LO term, OPEP plus Dirac- $\delta$ for ${ }^{3} S_{1}-{ }^{3} D_{1}$ and ${ }^{1} S_{0}$ and OPEP for the higher partial waves are brought from the fixed point at infinite to the reference scale $\mu^{2}$, where the physical information is supplied to the two-nucleon system. Moreover, through the RG equations we are able to evolve precisely the driving term of the subtracted equations to any arbitrary scale without altering the physical content of the observables. However, in this case the operator form of $V^{(n)}$ will acquire a nontrivial form and will be not easily identified with the starting potential.

\section{Full-on-shell amplitudes along the recursive renormalization process}

The next step is the computation of the full-on-shell matrices $V^{(n)}\left(k, k ; k^{2} ;-\mu^{2}\right)$, obtained from the solution of the recursive Eqs. (23) to (26), with $p=p^{\prime}=k$. Once we have the on-shell $T$ matrix, computed from Eq. (27) with $p=k$, we can obtain the $S$ matrix as

$$
S=1-2 i k T \text {. }
$$

Figures 5-7 present the full-on-shell recursive potentials, as we step forward in the recursive subtractive renormalization process, and the real part of $T\left(k, k ; k^{2}\right)$ [denoted by $T(k, k)$ in the figures] in the uncoupled states with total angular momentum up to $j=2$. For coupled channels, with $j=1$ and $j=2$, the evolution of the recursive potential and the real part of the scattering amplitude are shown in Figs. 8 and 9.

Results for ${ }^{1} S_{0}$ and ${ }^{3} P_{0}$ are shown in Fig. 5. Clearly, the results of scattering amplitude for the singlet $S$-wave channel are far from perturbative. At zero energy, a strong deviation of $T(0,0 ; 0)$ (that gives the scattering length) from $V^{(4)}\left(0,0 ; 0 ;-\mu^{2}\right)$ is observed, which is attributable to the
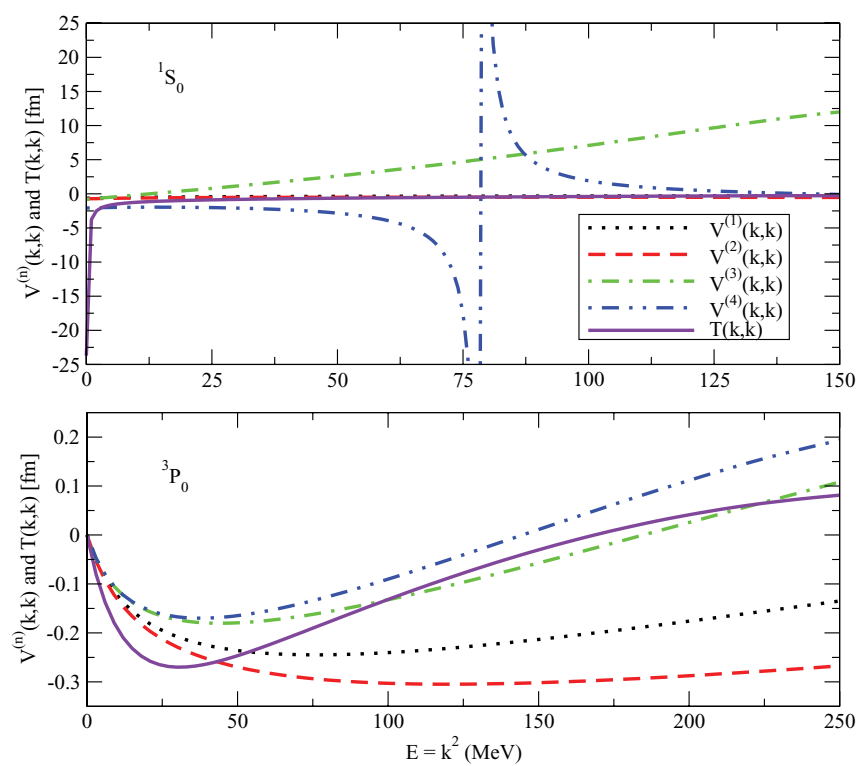

FIG. 5. (Color online) On-shell elements $V^{(n)}\left(k, k ; k^{2}\right)$ and $T\left(k, k ; k^{2}\right)$ for the uncoupled channels with $J=0$, as function of $k^{2}$. The legend for the curves, given in the top panel, is the same for both panels. 

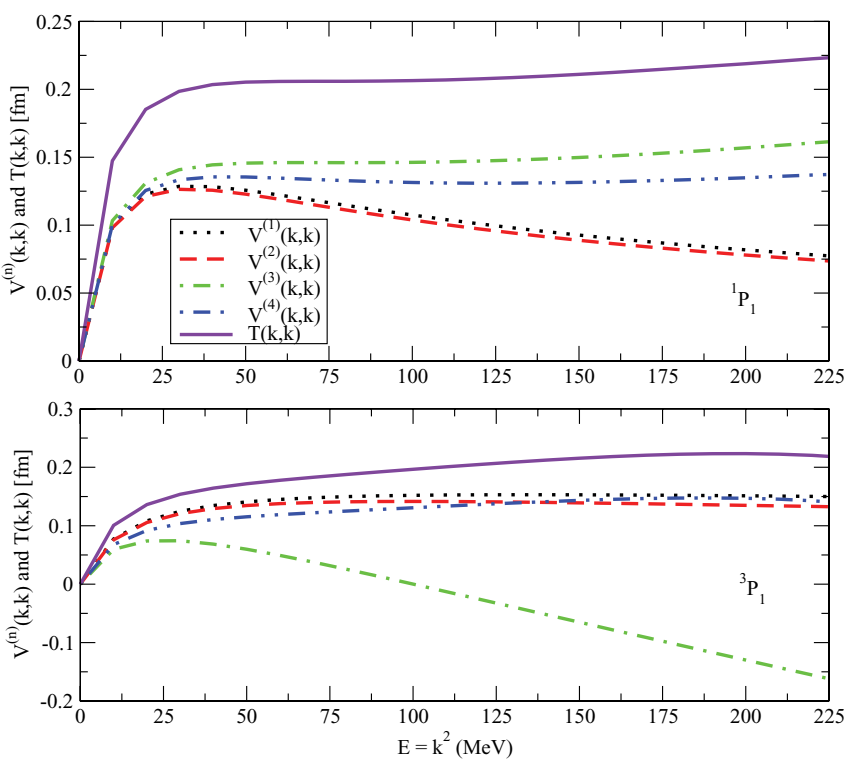

FIG. 6. (Color online) On-shell elements $V^{(n)}\left(k, k ; k^{2}\right)$ and $T\left(k, k ; k^{2}\right)$ for the uncoupled channels with $J=1$, as function of $k^{2}$. The legend for the curves, given in the top panel, is the same for both panels.

nearby singlet virtual state. The contribution of the contact in NLO to $V^{(3)}\left(k, k ; k^{2} ;-\mu^{2}\right)$ distinguishes it from the recursive process. In particular, $V^{(4)}\left(k, k ; k^{2} ;-\mu^{2}\right)$ presents a pole, which arises from the solution of the integral equation, which does not affect the scattering amplitude in the energy range that is shown. The comparison of $V^{(4)}\left(k, k ; k^{2} ;-\mu^{2}\right)$ with the real part of $T\left(k, k ; k^{2}\right)$ for ${ }^{3} P_{0}$ shows a behavior that indicates the dominance of the driven term. The zero of both quantities are close together and the magnitude of the real part of $T\left(k, k ; k^{2}\right)$
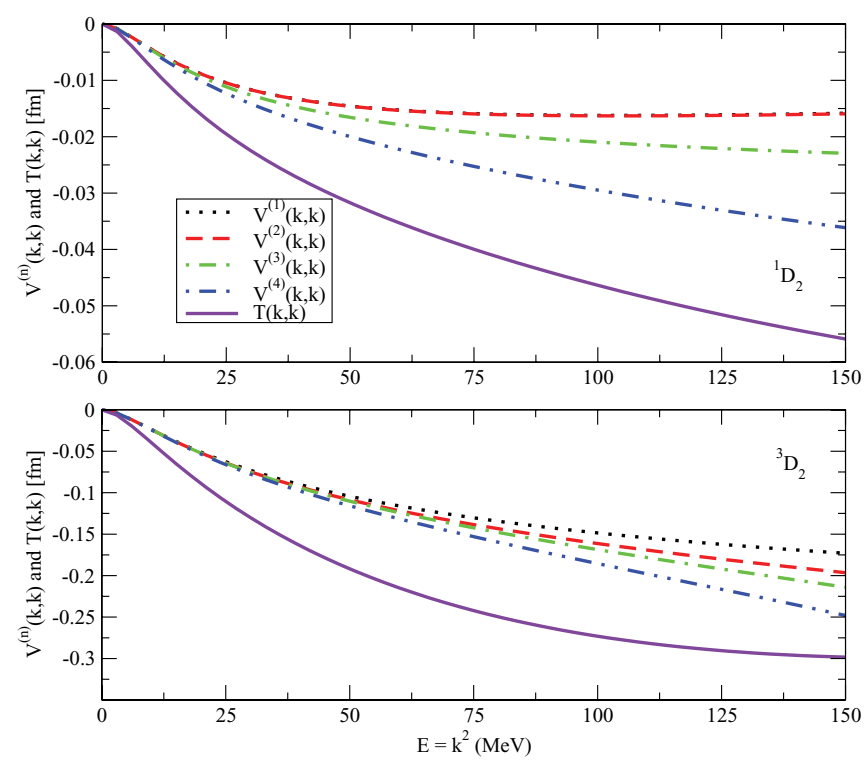

FIG. 7. (Color online) On-shell elements $V^{(n)}\left(k, k ; k^{2}\right)$ and $T\left(k, k ; k^{2}\right)$ for the uncoupled channels with $J=2$, as function of $k^{2}$. The legend for the curves, given in the top panel, is the same for both panels.

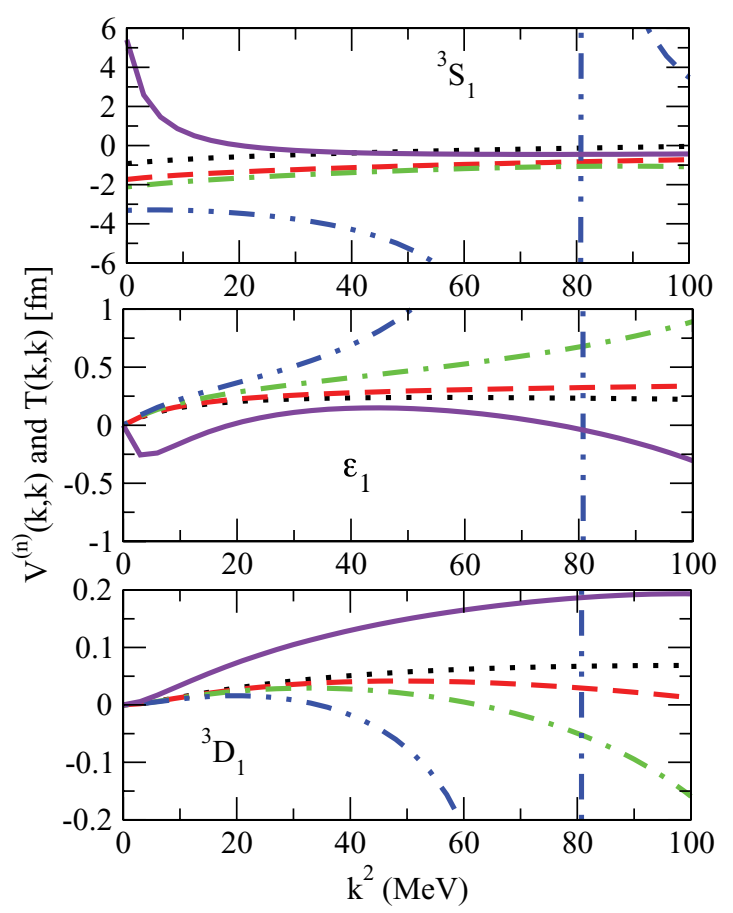

FIG. 8. (Color online) On-shell elements $V^{(n)}\left(k, k ; k^{2}\right)$ and $T\left(k, k ; k^{2}\right)$ for the coupled channels with $J=1$. In the middle panel, the amplitude $T\left(k, k ; k^{2}\right)$ for $\epsilon_{1}$ has been multiplied by a factor of 10 to highlight its qualitative behavior. The legend for the curves appearing in the three panels is the same as that given in Fig. 7.

decreases in respect to $V^{(4)}\left(k, k ; k^{2} ;-\mu^{2}\right)$ with energy. The contribution of the NLO contact to $V^{(3)}\left(k, k ; k^{2} ;-\mu^{2}\right)$ is also clearly seen in the figure, while the TPE NNLO potential appears to be not so much relevant in this wave.

The on-shell matrix elements of the recursive potential for ${ }^{1} P_{1}$ and ${ }^{3} P_{1}$ and scattering amplitude are shown in Fig. 6. For these waves the TPE NNLO potential does not gives a relevant contribution as one observe from the difference between $V^{(4)}\left(k, k ; k^{2} ;-\mu^{2}\right)$ and $V^{(3)}\left(k, k ; k^{2} ;-\mu^{2}\right)$. The NLO contact is important in these waves, as also seen for ${ }^{3} P_{0}$. The real part of scattering amplitude for ${ }^{1} P_{1}$ is also somewhat dominated by $V^{(4)}\left(k, k ; k^{2} ;-\mu^{2}\right)$. The uncoupled $D$ waves are shown in Fig. 7. No contacts are present and the recursive potentials change smoothly from one to the next.

The coupled ${ }^{3} S_{1}-{ }^{3} D_{1}$ on-shell potentials and scattering amplitudes are presented in Fig. 8. The real part of the scattering amplitudes for these channels are far from perturbative. At zero energy the real part of $t(0,0 ; 0)$ for the ${ }^{3} S_{1}$ channel, which gives the scattering length, moves strongly from $V^{(4)}\left(0,0 ; 0 ;-\mu^{2}\right)$ owing to the deuteron pole. The same is observed at low energies for the off-diagonal amplitude, related to the importance of the deuteron $D / S$ ratio to the mixing parameter [42]. The contribution of the contact in NLO to $V^{(3)}\left(k, k ; k^{2} ;-\mu^{2}\right)$ distinguishes it from the recursive process. The pole of $V^{(4)}\left(k, k ; k^{2} ;-\mu^{2}\right)$ does not affect the scattering amplitude in the energy range of our calculations. The coupled ${ }^{3} P_{2}-{ }^{3} F_{2}$ on-shell potentials and scattering amplitudes are seen in Fig. 9, and we observe a strong increase in the magnitude 


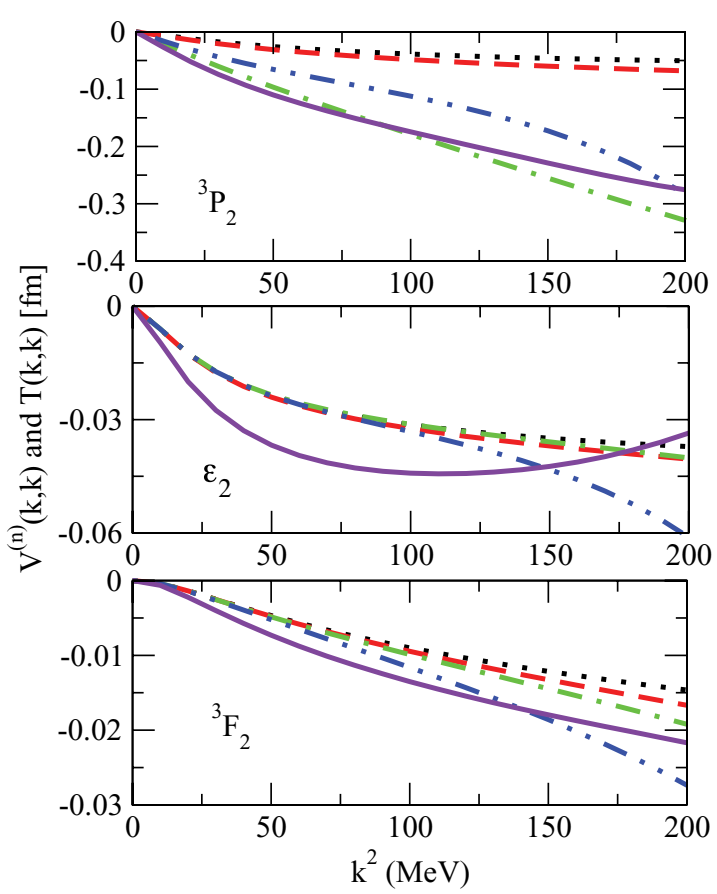

FIG. 9. (Color online) On-shell $V^{(n)}\left(k, k ; k^{2}\right)$ and $T\left(k, k ; k^{2}\right)$ for the coupled channels with $J=2$. The legend for the curves appearing in the three panels is the same as the one given in Fig. 7.

of $V^{(4)}\left(k, k ; k^{2} ;-\mu^{2}\right)$, which also is an indication of the contribution of the NNLO potential to this wave.

\section{RESULTS FOR NUCLEON-NUCLEON PHASE SHIFTS AND MIXING PARAMETERS}

For the analysis of the phase shifts and mixing parameters obtained with the renormalized strengths and subtraction energies presented in Tables I to IV, we adopt a systematics which splits the calculations in four sets:

(i) LO, as given in Eq. (17);

(ii) LO plus NLO contact interactions ( $\mathrm{LO}+\mathrm{NLO} \mathrm{CI})$, as given in Eq.(19);

(iii) full NLO, consisting of one-pion exchange, two-pion exchange at NLO, and contact interactions;

(iv) NNLO, which is the NLO plus TPE diagrams at NNLO.

In particular, set (ii) was inspired by the idea to promote some NLO terms to LO as a way to overcome difficulties with the Weinberg power-counting rule (see Ref. [3]). One consequence of the failure of the NDA is that contact interactions that are subleading in Weinberg's power conting are, in fact, necessary to make sense of the $T$ matrix even at LO. In Refs. [2-4], it is shown how the origin of the failure of NDA is the singularity of one pion exchange (OPE), for which a single counterterm suffices at LO.

For the LO potential in the singlet and triplet channels, the respective scattering lengths are fitted. Indeed this calculation reproduces the results obtained in Ref. [5], which we supply here for completeness and to compare with the results obtained in NLO and NNLO. The results for the phase shifts for the
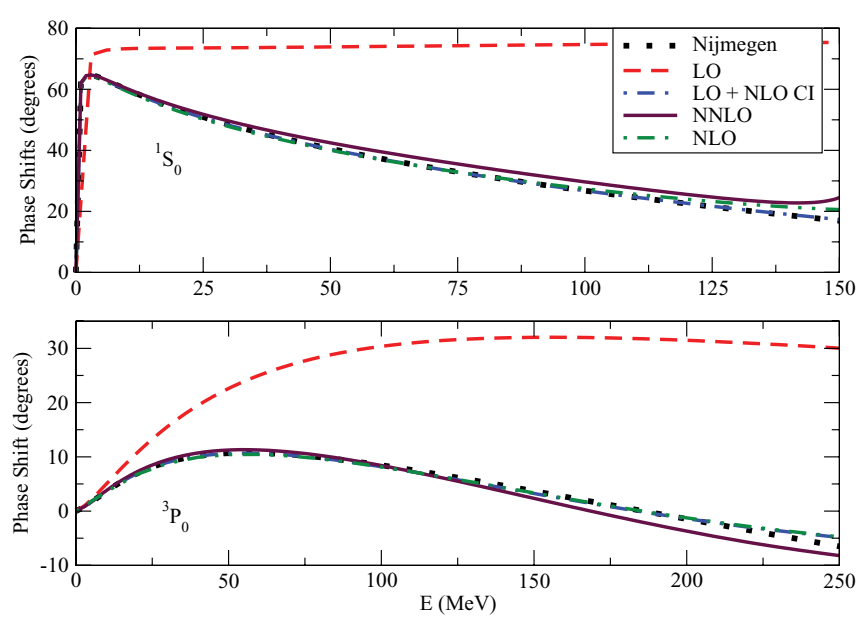

FIG. 10. (Color online) Uncoupled channels for $J=0$. Phase shifts for the ${ }^{1} S_{0}$ wave with the subtraction point at $-50 \mathrm{MeV}$ and for the ${ }^{3} P_{0}$ wave with the subtraction point at $-100 \mathrm{MeV}$. The legend for the curves, given in the top panel, is the same for both panels.

waves ${ }^{1} S_{0}$ and ${ }^{3} P_{0}$ are shown in Fig. 10 . We should observe that results corresponding to the LO + NLO CI were already presented in Ref. [10]. The NLO and NNLO calculations were done by using the same renormalized strengths for the derivative of the contacts (see $\lambda_{2}$ and $\lambda_{3}$ in Tables II-IV) and only refitted the singlet scattering length by changing slightly $\lambda_{0}$. This means that at the range of energies we perform our calculations the contributions of TPE potentials in this wave are small. Moreover, the NLO and NNLO calculations present a small systematic deviation steadily increasing with energy. This is possibly attributable to the strong attraction of the corresponding TPE NLO and NNLO potentials which increases at higher momentum.

In the ${ }^{3} P_{0}$ shown in the bottom panel of Fig. 10 there is a contact interaction of the type $V_{\text {contact }}=\lambda_{1} p p^{\prime}$ at NLO along with one-pion and two-pion exchanges. The LO calculation is not able to fit the scattering volume of ${ }^{3} P_{0}$, while with $\lambda_{1}$ given in Tables II to IV we obtain a better fit as shown by the corresponding adjustment of the low-energy Nijmegen phase shifts. It is seen that a small change in $\lambda_{1}$ is enough to give the scattering volume for sets (ii), (iii), and (iv). The small change in the contact term which fits the singlet scattering length is similar to what is found for ${ }^{3} P_{0}$ in respect to the scattering volume.

The phase shifts for the waves ${ }^{1} P_{1}$ and ${ }^{3} P_{1}$ are shown in Fig. 11. In both channels there is a contact interaction of the type $V_{\text {contact }}=\lambda_{1} p p^{\prime}$ at NLO along with one-pion and twopion exchanges. In this case, we observe that the LO + NLO $\mathrm{CI}$ is already giving a quite good fit, when comparing with the Nijmegen results. The TPEP contribution is marginal as observed by the slight change of $\lambda_{1}$ required to keep the fit (see Tables II to IV).

The results for the phase shifts for the uncoupled channels ${ }^{1} D_{2}$ and ${ }^{3} D_{2}$ are shown in Fig. 12. As already mentioned, no contact terms are presented, in this case.

In the following, we discuss the results for the coupled channels for the spin triplet $j=1$ in the ${ }^{3} S_{1}-{ }^{3} D_{1}$ states and $j=2$ in the ${ }^{3} P_{2}-{ }^{3} F_{2}$ states. The phase shifts for the ${ }^{3} S_{1}-{ }^{3} D_{1}$ 

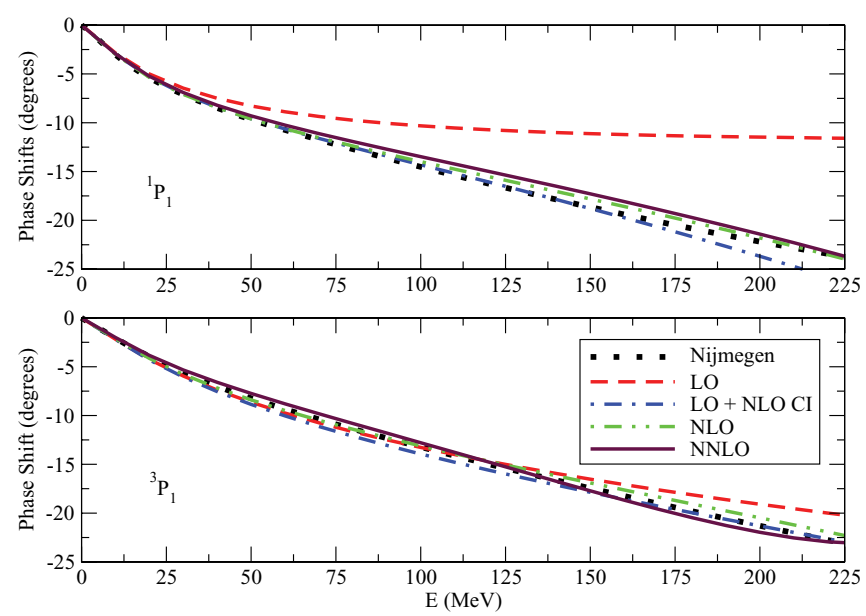

FIG. 11. (Color online) Phase shifts for the ${ }^{1} P_{1}$ and ${ }^{3} P_{1}$ waves. The legend for the curves is given in the bottom panel for both the cases.

coupled channels are shown in Fig. 13 and for ${ }^{3} P_{2}-{ }^{3} F_{2}$ in Fig. 14.

For the mixing parameter ${ }^{3} S_{1}-{ }^{3} D_{1}$, we consider $\epsilon_{1}$, as defined in Ref. [43], instead of the Blatt-Biedenharn definition, $\epsilon_{B B}$ [44]. The reason for this choice is related to the precise measurements available for $\epsilon_{1}$. We observe in Fig. 13 that the mixing parameter $\epsilon_{1}$ can be well fitted with only the LO plus a small contact term in the mixed states (see $\lambda_{4}$ in Table II). This indicates that the physics of $\epsilon_{1}$ seems well controlled by OPEP, as long ago predicted by a shape-independent expansion [42].

What do we see when the NLO and NNLO potentials are inserted in our method? The aforementioned nice fitting of $\epsilon_{1}$ by the LO potential plus small contact term in the mixed channel disappears (see Fig. 13). We met the widely recognized difficulty that the effective potential has problems in the describing $\epsilon_{1}$. To make concrete this point we mention that different renormalization approaches, coordinate space renormalization method [30], and subtraction plus cutoff [34] also faced the same difficulty to fit $\epsilon_{1}$ in NLO and NNLO.
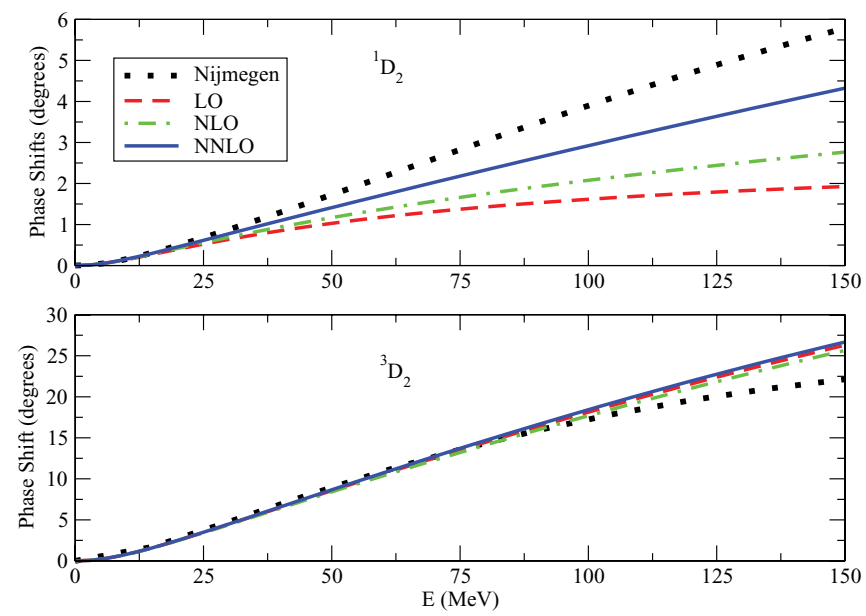

FIG. 12. (Color online) Phase shifts for the ${ }^{1} D_{2}$ and ${ }^{3} D_{2}$ waves. The legend for the curves is given in the top panel for both the cases.
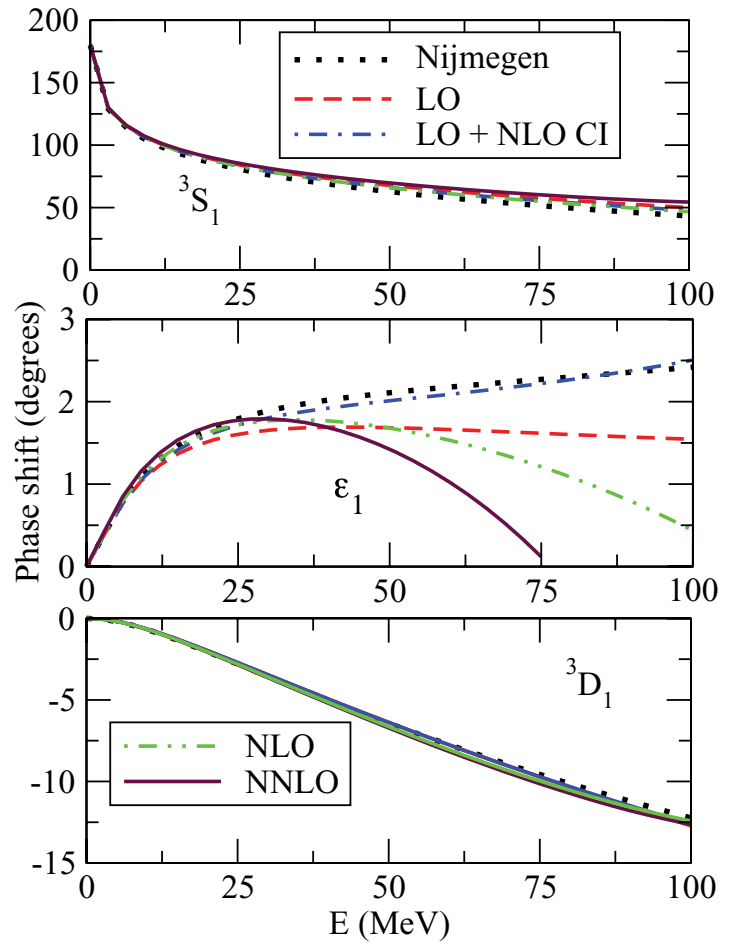

FIG. 13. (Color online) Phase shifts and mixing parameter $\epsilon_{1}$ for the ${ }^{3} S_{1}-{ }^{3} D_{1}$ coupled channels. The legend for the curves appearing in the three panels is shown in the top and bottom panels.

In particular, Ref. [34] exploited the strong momentum cutoff dependence to fit $\epsilon_{1}$; however, the fit is not robust in the sense that it should be smoothly cutoff dependent. This common difficulty in different and independent calculations reveals the distinct role played by the singularities in different waves.

From the results shown in Fig. 13, we see that the TPEP does not exhibit a systematic behavior in the different partial waves regarding the NLO and NNLO potentials. From our point of view a systematic behavior would require a cutoff for TPEP or its inclusion only after higher-order singular terms are included. This problem is acute in the mixing parameter of the coupled channel ${ }^{3} S_{1}-{ }^{3} D_{1}$. The mixing parameter with OPEP plus NLO contact is well fitted up to $100 \mathrm{MeV}$ laboratory energy. The introduction of NLO and NNLO TPEP clearly worsens the fit to the Nijmegen phase-shift analysis [45]. To fit the mixing parameter it is important to have the deuteron asymptotic $D / S$ ratio within their accepted values. This was pointed out in a recent work [31], where the authors have also included the contribution of the $\delta$ excitation. Indeed, long ago, it was already concluded that the mixing parameter at low energies is determined by the deuteron properties and by OPEP [42]. By considering the Blatt-Biedenharn [44] definition, it was demonstrated in [42] that the correct long-range behavior of the tensor potential is essential for a realistic reproduction of the mixing parameter. As shown in Ref. [42], separable tensor Yamaguchi and square-well potentials, which do not possess the OPE tail, when fitted to reproduce the deuteron binding and asymptotic normalization, badly fail to reproduce the correct value of the mixing parameter. The NLO and NNLO potentials for the deuteron channel seem to give a too strong contribution, 

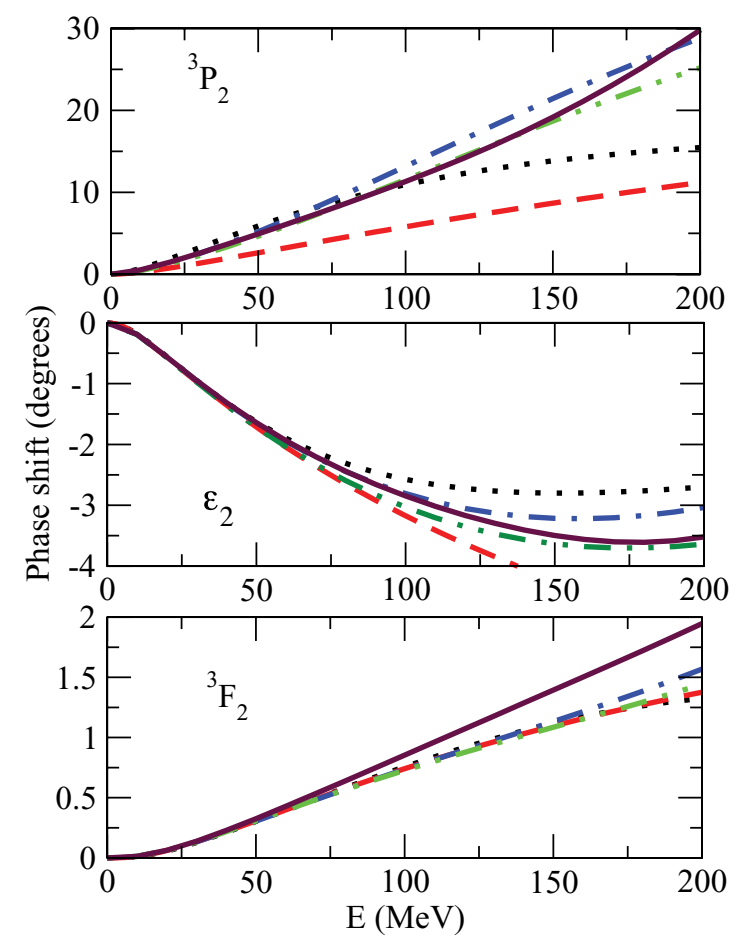

FIG. 14. (Color online) Phase shifts and mixing parameter $\epsilon_{2}$ for the ${ }^{3} P_{2}-{ }^{3} F_{2}$ coupled channels. The legend for the curves appearing in the three panels is the same as that given in Fig. 13.

enhanced by the singular behavior of OPEP at short distances in this channel. Although the diagonal and off-diagonal NLO contact plus OPEP and LO contact are enough to give a nice fitting to the mixing parameter up to $E_{\text {lab }}=100 \mathrm{MeV}$, the inclusion of NLO and NNLO TPEP does not provide a good fit, and the results systematically deviate from the Nijmegen data for $\epsilon_{1}$. This indicates that by keeping the OPEP potential intact, the NLO and NNLO TPEP potentials have to have a cut at short distances.

It is appropriate to summarize the comparison between our method of multiple subtractions and that used in Ref. [34]. In ours no cutoff is needed while in the latter one just one subtraction is required because higher order singularities coming from the $\delta$ derivatives are tamed by a cutoff. Numerically, in general, both procedures lead to a similar fitting for the $S$ and $P$ waves up to energies of about $200 \mathrm{MeV}$. We also remark that the mixing parameter, $\epsilon_{1}$, presents the same deficiency in the fit by both methods, as well as for a different regularization and renormalization method in coordinate space without the contribution of the $\delta$ excitation [31].

We note that, once the parameters are fitted (renormalized strengths and subtraction points) the subtraction scale can be moved without changing the $T$-matrix results. Our method is RG invariant by construction. The flow equation (8), which transforms the driven term of the subtracted scattering equation (27) under the dislocation of the subtraction point, was derived from the invariance of the scattering matrix under variations of $\mu$.

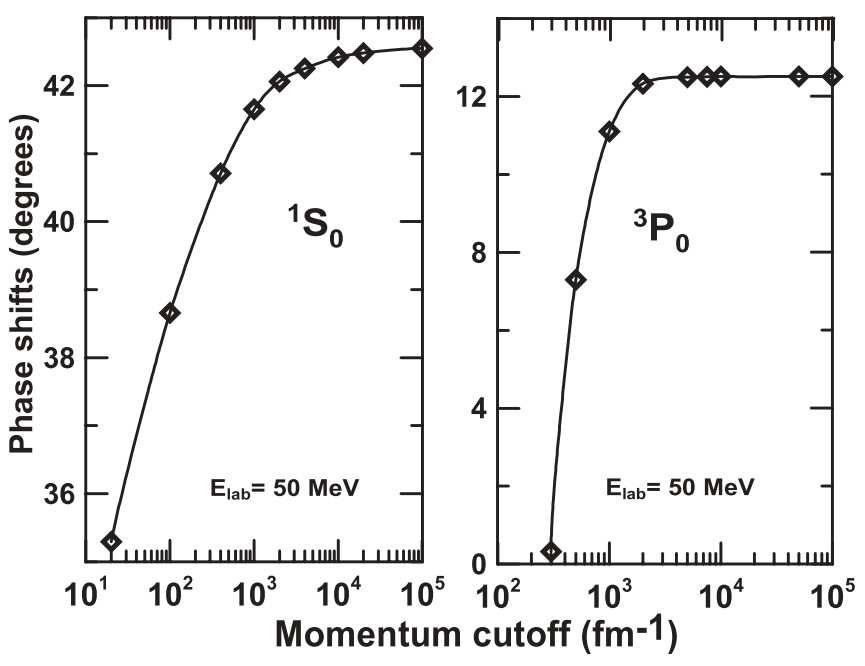

FIG. 15. Phase shifts for the ${ }^{1} S_{0}$ (left) and ${ }^{3} P_{0}$ (right) waves, for $E_{\text {lab }}=50 \mathrm{MeV}$, exemplifying a general behavior of the phase shifts for increasing cutoffs. Both the cases presented here were calculated considering the NNLO potential, using the $n=4$ subtracted scattering equation (27), with parameters given in Table IV. The same kind of behavior is verified for the other orders of the potential (LO and NLO), as well as for other waves and $E_{\text {lab }}$ energies.

To conclude this section, we illustrate the cutoff independence of our method. We choose to present results for two singular cases, the ${ }^{1} S_{0}$ and ${ }^{3} P_{0}$ phase shifts at $50 \mathrm{MeV}$. They were obtained by considering the NNLO potential, with $n=4$ subtracted scattering equation (27). As shown in Fig. 15, the phase shifts stabilize when the cutoffs are driven toward infinity. The same kind of cutoff behavior is verified for the other orders of the potential (LO and NLO), as well as for other partial waves and $E_{\text {lab }}$ energies. Our results shown in this paper were obtained for infinite cutoffs, with the verified convergence owing to the subtractive renormalization approach we are considering.

\section{CONCLUSION}

We present a systematic methodology to renormalize the nucleon-nucleon interaction using a recursive subtracted approach with multiple subtractions in the kernel. Within the subtractive scheme we studied the two-nucleon scattering $T$ matrix for the NLO and NNLO potentials for all partial waves up to $j=2$. The renormalized strengths of the contact interactions, the so-called low-energy constants, were fitted to the low-energy phase-shifts and mixing parameters from the Nijmegen partial wave analysis for a reference subtraction point.

To show how the multiple subtraction renormalization scheme can be implemented, an analytical example is given for the $P$-wave channel. In that case, two subtractions are required to eliminate the ultraviolet singularity of the interaction. We give explicitly the solution of the RG equation. Although we have not fully explored the analytical form of this $P$-wave amplitude, we call the attention of the reader to the richness 
of the analytical continuation to the complex energy plane to obtain virtual and resonant states [37,46,47].

In a sharp or smooth cutoff approach, the LO, NLO, and NNLO potentials are regularized to vanish above a certain momentum scale and then inserted in the LS equation. In our method, the cutoff is purely instrumental and the limit of the momentum cutoff going to infinity can be easily performed, because a finite $T$ matrix is ensured by multiple subtractions in the scattering equation. The original potential is kept intact and enters in the recursive process, as we described in detail.

We analyze the matrix elements of the potentials in momentum space for the ${ }^{1} S_{0}$ channel as we go from LO through NLO up to NNLO. This suggests in a practical way a momentum scale, which we associate with the subtraction point, where the systematic expansion of the potential from the chiral EFT should be used within our subtraction scheme. From such analysis, we verify that a momentum scale of about $1 \mathrm{fm}^{-1}$ separates the matrix elements of NLO and NNLO potentials in two regions: Below this scale they are comparable to the OPEP. With this indication, we choose a subtraction point at an energy $-\mu^{2} \sim-50$ to $-100 \mathrm{MeV}$. The subtraction momentum scale comes to be at the order of the QCD scale, $\Lambda_{\mathrm{QCD}}[48]$, and well below the $\rho$-meson mass, consistent with the general suggestion of Weinberg [1]. It is important to observe that, by taking into account the values of the $N N$ low-energy parameters and the form of the bare interaction including the derivatives of the contacts, a renormalization scale near the value of the $\Lambda_{\mathrm{QCD}}$ is consistent with the Wigner bound $[49,50]$. Once we fit the renormalized strengths of the contacts, considering the pion exchange potentials at LO, NLO, and NNLO, the subtraction point can be moved arbitrarily. Our method is RG invariant by construction, and the flow equation [Eq. (8)] transforms the driven term of the subtracted scattering equation such that scattering matrix is invariant under dislocation of $\mu$.

We show how the half-on-shell potentials for ${ }^{1} S_{0}$ and ${ }^{3} S_{1}$ channels evolve through the four subtractions, from $V^{(1)}(q, k)$ up to $T\left(q, k ; k^{2}\right)$. These exhibit a relation with the interesting finding of Redish-Stickbauer [41] that the half-on-shell potential and corresponding half-on-shell $T$ matrix can be very different. Given distinct $V(q, k)$, which fit the same on-shell observables, the corresponding half-on-shell $T$ matrix should be quite equivalent in spite the sharp $V(q, k)$ differences. That is consistent with the smooth behavior of the scattering amplitude with energy, while the recursive driving terms can vary considerably.

In the ${ }^{3} P_{0}$ channel, a contact interaction is introduced at NLO together with OPEP and TPEP. The LO calculation is not able to fit the scattering volume of ${ }^{3} P_{0}$, while the addition of contact gives a better fit of the Nijmegen phase shifts. It is verified that a small change in the renormalized strength of the contact, obtained only with OPEP, is enough to reproduce the scattering volume for NLO and NNLO. The same behavior is observed for the strength of the contact interaction when the singlet scattering length is fitted with NLO and NNLO potentials.

For the ${ }^{1} P_{1}$ and ${ }^{3} P_{1}$ channels we introduce a contact interaction at NLO along with OPEP and TPEP. A quite good fit of the Nijmegen phase shifts is obtained with OPEP plus the contact. Again we observe that the TPEP contribution is marginal as a slight change of the renormalized strengths keeps the fit. These observations indicate the absence of an essential singularity in the OPEP and TPEP potentials up to NNLO in uncoupled $P$ waves, beyond the contacts itself, differently from what is found for the coupled ${ }^{3} S_{1}-{ }^{3} D_{1}$ channel.

After our fitting of the contact interactions for the $P$ waves $\left({ }^{3} P_{0},{ }^{3} P_{2},{ }^{1} P_{1},{ }^{3} P_{1}\right)$, the following observations apply to the matrix elements in momentum space of the LO, NLO, and NNLO potentials. For ${ }^{3} P_{0}$ and ${ }^{1} P_{1}$, the contact dominates above $3 \mathrm{fm}^{-1}$, while below $2 \mathrm{fm}^{-1}$ the LO potential dominates. For the ${ }^{3} P_{2}$, the LO potential is weak, while the inclusion of the contact interaction enhances the attraction of the NLO potential, which weakens the strong repulsion from the NNLO TPEP.

Next we summarize our findings for the coupled channels: ${ }^{3} S_{1}-{ }^{3} D_{1}$ and ${ }^{3} P_{2}-{ }^{3} F_{2}$. The mixing parameter $\epsilon_{1}$ can be well fitted at LO plus a small contact term which couples the $S-D$ states in the interaction. This indicates that the physics of $\epsilon_{1}$ seems well controlled by OPEP, as suggested in Ref. [42] by a shape-independent expansion. When we include the NLO and NNLO potentials within our method, the nice fitting $\epsilon_{1}$ is destroyed. The NLO and NNLO potentials for the deuteron channel seem to give a too strong contribution, enhanced by the singular behavior of the OPEP at short distances in this channel.

The difficulty in fitting $\epsilon_{1}$ with effective potentials in NLO and NNLO is recognized by different renormalization approaches $[30,34]$. Such a shortcoming is related to the strong momentum cutoff dependence of $\epsilon_{1}$, as found, for example, by the coordinate renormalization approach [30], possibly owing to the singular behavior of OPEP [35].

The contribution of TPEP does not exhibit a systematic behavior in the different partial waves regarding the NLO and NNLO potentials. Our results suggest that, for a systematic behavior, the TPEP should be weakened for momentum larger than few $\mathrm{fm}^{-1}$. This could be done via a cutoff or by considering higher order contacts, which could suppress the TPEP contribution in the appropriate partial waves.

We found that the derivative contact terms dominate over the NLO and NNLO two-pion exchange interactions in the $S$-wave channels, starting at low-momentum scales $\left(\sim 0.3 \mathrm{fm}^{-1}\right)$. In $P$-wave channels the contacts are also important for the fitting of the corresponding phase shifts.

Finally, we should observe that the input of our method is the $T$ matrix at a given energy, where the physical information is supplied to the two-nucleon system. When the interaction is at LO, the point where the physical input is given is not constrained. Once we move to NLO and NNLO, the energy $-\mu^{2}$ arises as a scale where the low-energy observables can be obtained. In view of that, the particular value of the subtraction point acquires the status of a physical scale when the NLO and NNLO interactions are introduced. It is gratifying to verify that our fittings with the associated subtraction point, given by the renormalization scale, comes to be about $\Lambda_{\mathrm{QCD}}$, well 
below the $\rho$-meson mass. This is consistent with the general Weinberg [1] suggestion that an effective potential should be valid for momenta much smaller than a typical QCD scale of $1 \mathrm{GeV}$, and the intermediate nucleon-nucleon propagation should be damped at such small momentum scale.

\section{ACKNOWLEDGMENTS}

We thank the Brazilian agencies Fundação de Amparo à Pesquisa do Estado de São Paulo (FAPESP) and Conselho Nacional de Desenvolvimento Científico e Tenológico (CNPq) for partial support. V.S.T. would like to thank FAEPEX/UNICAMP for partial support.

\section{APPENDIX: $N \boldsymbol{N}$ PHASE SHIFTS AND MIXING PARAMETERS}

We follow the definitions given in Ref. [43] for the nucleon-nucleon phase shifts and mixing parameters, which are appropriate to the case where we have coupled channels. Considering the cases with $j>0$, for the coupled channels we have an $S$-matrix expression given by

$$
\begin{aligned}
(S)_{j} \equiv & \left(\begin{array}{ll}
S_{j-1, j-1} & S_{j-1, j+1} \\
S_{j+1, j-1} & S_{j+1, j+1}
\end{array}\right)=\left(\begin{array}{cc}
e^{\mathrm{i} \delta_{j-1}} & 0 \\
0 & e^{\mathrm{i} \delta_{j+1}}
\end{array}\right) \\
& \times\left(\begin{array}{cc}
\cos \left(2 \epsilon_{j}\right) & \mathrm{i} \sin \left(2 \epsilon_{j}\right) \\
\mathrm{i} \sin \left(2 \epsilon_{j}\right) & \cos \left(2 \epsilon_{j}\right)
\end{array}\right)\left(\begin{array}{cc}
e^{\mathrm{i} \delta_{j-1}} & 0 \\
0 & e^{\mathrm{i} \delta_{j+1}}
\end{array}\right),
\end{aligned}
$$

from where the corresponding phase shifts and mixing parameter are

$$
\begin{aligned}
\delta_{j-1} & =\frac{1}{2} \tan ^{-1}\left(\frac{\operatorname{Im} S_{j-1, j-1}}{\operatorname{Re} S_{j-1, j-1}}\right), \\
\delta_{j+1} & =\frac{1}{2} \tan ^{-1}\left(\frac{\operatorname{Im} S_{j+1, j+1}}{\operatorname{Re} S_{j+1, j+1}}\right), \\
\epsilon_{j} & =-\frac{1}{2} \tan ^{-1}\left(\frac{\mathrm{i}\left(S_{j-1, j+1}+S_{j+1, j-1}\right)}{2 \sqrt{S_{j-1, j-1} S_{j+1, j+1}}}\right) .
\end{aligned}
$$

and L. Tomio, Few-Body Syst. 28, 259 (2000); T. Frederico, A. Delfino, and L. Tomio, ibid. 31, 235 (2002); M. T. Yamashita, L. Tomio, A. Delfino, and T. Frederico, Euro Phys. Lett. 75, 555 (2006); L. Tomio, Few-Body Syst. 43, 207 (2008); M. T. Yamashita, T. Frederico, and L. Tomio, ibid. 44, 191 (2008).

[13] M. C. Birse, Phys. Rev. C 74, 014003 (2006).

[14] C. Ordóñez, L. Ray, and U. van Kolck, Phys. Rev. C 53, 2086 (1996).

[15] G. P. Lepage, How to Renormalize the Schrödinger Equation, Proceedings of the VIII Jorge André Swieca Summer School Nuclear Physics, edited by C. A. Bertulani, M. E. Bracco, B. V. Carlson, and M. Nielsen (World Scientific, Singapore, 1997), p. 135.

[16] N. Kaiser, R. Brockmann, and W. Weise, Nucl. Phys. A 625, 758 (1997); N. Kaiser, S. Gerstendorfer, and W. Weise, ibid. 637, 395 (1998).

[17] J. L. Friar, Phys. Rev. C 60, 034002 (1999).

[18] P. F. Bedaque and U. van Kolck, Annu. Rev. Nucl. Part. Sci. 52, 339 (2002).

[19] E. Epelbaum, Prog. Part. Nucl. Phys. 57, 654 (2006).

[20] E. Epelbaum, H.-W. Hammer, and Ulf-G. Meissner, Rev. Mod. Phys. 81, 1773 (2009); E. Epelbaum and J. Gegelia, Eur. Phys. J. A 41, 341 (2009).

[21] D. B. Kaplan, M. J. Sawage, and M. D. Wise, Phys. Lett. B 424, 390 (1998).

[22] M. C. Birse, J. A. McGovern, and K. G. Richardson, Phys. Lett. B 464, 169 (1999); T. Barford and M. C. Birse, Phys. Rev. C 67, 064006 (2003); M. C. Birse and J. A. McGovern, ibid. 70, 054002 (2004).

[23] T.-S. Park, K. Kubodera, D.-P. Min, and M. Rho, Nucl. Phys. A 646, 83 (1999); Phys. Rev. C 58, R637 (1998).

[24] T. Mehen and I. W. Stewart, Phys. Lett. B 445, 378 (1999); S. Fleming, T. Mehen, and I. W. Stewart, Nucl. Phys. A 677, 313 (2000).

[25] R. J. Perry and S. Szpigel, A new renormalization group for Hamiltonian Field Theory, arXiv:nucl-th/9901079.

[26] D. R. Entem and R. Machleidt, Phys. Rev. C 68, 041001 (2003). 
[27] K. A. Scaldeferri, D. R. Phillips, C.-W. Kao, and T. D. Cohen, Phys. Rev. C 56, 679 (1997); T. D. Cohen and J. M. Hansen, ibid. 59, 13 (1999); 59, 3047 (1999).

[28] J. Gegelia, Phys. Lett. B 429, 227 (1998); J. Phys. G 25, 1681 (1999); J. Gegelia and S. Scherer, Int. J. Mod. Phys. A 21, 1079 (2006).

[29] J. L. Ballot and M. R. Robilotta, J. Phys. G 20, 1599 (1994).

[30] M. P. Valderrama and E. R. Arriola, Phys. Rev. C 70, 044006 (2004); 72, 054002 (2005); M. Pavon Valderrama and E. Ruiz Arriola, ibid. 74, 054001 (2006); M. P. Valderrama and E. R. Arriola, Ann. Phys. 323, 1037 (2008).

[31] M. P. Valderrama and E. R. Arriola, Phys. Rev. C 79, 044001 (2009).

[32] D. R. Entem and E. R. Arriola, Phys. Rev. C 80, 047001 (2009).

[33] C.-J. Yang, Ch. Elster, and D. R. Phillips, Phys. Rev. C 77, 014002 (2008).

[34] C.-J. Yang, Ch. Elster, and D. R. Phillips, Phys. Rev. C 80, 034002 (2009).

[35] B. Long and U. van Kolck, Ann. Phys. 323, 1304 (2008).

[36] M. Pavon Valderrama, Phys. Rev. C 83, 024003 (2011).

[37] C. A. Bertulani, H.-W. Hammer, and U. van Kolck, Nucl. Phys. A 712, 37 (2002).

[38] S. Weinberg, The Quantum Tehory of Fields (Cambridge University Press, Cambridge, 1996).
[39] S. K. Bogner, T. T. S. Kuo, and A. Schwenk, Phys. Rep. 386, 1 (2003).

[40] K. Harada, H. Kubo, and A. Ninomiya, Int. J. Mod. Phys. A 24, 3191 (2009).

[41] E. F. Redish and K. Stricker-Bauer, Phys. Rev. C 36, 513 (1987).

[42] S. K. Adhikari, L. Tomio, J. P. B. C. de Melo, and T. Frederico, Phys. Lett. B 318, 14 (1993); S. K. Adhikari, C. F. de Araujo, and T. Frederico, Phys. Rev. C 50, R2684 (1994).

[43] H. P. Stapp, T. J. Ypsilantis, and N. Metropolis, Phys. Rev. 105, 302 (1957).

[44] J. M. Blatt and L. C. Biedenharn, Phys. Rev. 86, 399 (1952); L. C. Biedenharn and J. M. Blatt, ibid. 93, 1387 (1954).

[45] V. G. J. Stoks, R. A. M. Klomp, C. P. F. Terheggen, and J. J. deSwart, Phys. Rev. C 49, 2950 (1994); V. G. J. Stoks, R. A. M. Klomp, M. C. M. Rentmeester, and J. J. deSwart, ibid. 48, 792 (1993).

[46] L. A. L. Roriz and A. Delfino, Phys. Rev. C 38, 607 (1988).

[47] A. Delfino and W. Glöckle, Phys. Rev. C 30, 376 (1984).

[48] C. Amsler et al. (Particle Data Group), Phys. Lett. B 667, 1 (2008).

[49] D. R. Phillips and T. D. Cohen, Phys. Lett. B 390, 7 (1997).

[50] S. Szpigel, V. S. Timóteo, and F. de O. Durães, Ann. of Phys. 326, 364 (2011). 